\title{
A SYSTEMATIC SEARCH FOR PERIODICITIES IN RXTE ASM DATA
}

\author{
Linging Wen, ${ }^{1}$ Alan M. Levine, ${ }^{2}$ Robin H. D. Corbet, ${ }^{3}$ and Hale V. Bradt ${ }^{2}$ \\ Received 2005 August 10; accepted 2005 November 22
}

\begin{abstract}
We present the results of a systematic search in $8.5 \mathrm{yr}$ of Rossi X-Ray Timing Explorer All-Sky Monitor data for evidence of periodicities. The search was conducted by application of the Lomb-Scargle periodogram to the light curves of each of 458 actually or potentially detected sources in each of four energy bands $(1.5-3,3-5,5-12$, and $1.5-12 \mathrm{keV})$. A whitening technique was applied to the periodograms before evaluation of the statistical significance of the powers. We discuss individual detections with a focus on relatively new findings.
\end{abstract}

Subject headings: binaries: general $-\mathrm{X}$-rays: stars

On-line material: color figure

\section{INTRODUCTION}

Periodic modulation of the X-ray intensity has been observed in many X-ray binary systems (see a general discussion in White et al. 1995). The periods of the detected signals range from a few milliseconds (Wijnands \& van der Klis 1998) to hundreds of days (Delgado-Martí et al. 2001) and are, in general, associated with spin of a neutron star, binary orbital motion, or precession of a tilted accretion disk or other phenomenon in which the period usually exceeds the orbital period. In addition, very long quasi periods have been found to be associated with the outbursts of X-ray transients.

The periodicities attributable to anisotropic emission from rotating neutron stars are found mostly in high-mass X-ray binaries (HMXBs) and in a few low-mass X-ray binaries (LMXBs). The pulse periods range from a few milliseconds to at least tens of minutes and, possibly, hours (e.g., Hall et al. 2000).

The known orbital periods of LMXBs range from 0.19 to $398 \mathrm{hr}$, with the majority between $1 \mathrm{hr}$ and 2 days (White et al. 1995). The observed properties of the orbital modulation depend on the viewing angle. At low orbital inclination angles $\left(<70^{\circ}\right)$, $\mathrm{X}$-ray orbital periods are rarely observed. At intermediate inclinations, periodic dipping behavior and, in a few cases, brief eclipses of the X-rays by the companion may be observed. The dips may be caused by obscuration of materials splashed above the disk plane when the gas stream from the companion hits the accretion disk. For high-inclination systems $\left(>80^{\circ}\right)$, X-ray eclipses have been observed in several systems, but the number is smaller than would be expected if the systems simply consisted of a dwarf companion overflowing its Roche lobe and transferring material to a compact object via a thin accretion disk (Joss \& Rappaport 1979). To resolve this discrepancy, it has been proposed (Milgrom 1978) that the central X-ray source is hidden behind a thick accretion disk rim while the X-rays are scattered via a photoionized corona above the disk and can still be seen. The source thus appears extended and may be partially eclipsed periodically by the bulge at the disk rim formed by the impact of the accretion flow on the disk.

\footnotetext{
1 Max Planck Institut für Gravitationsphysik, Albert-Einstein-Institut, Am Mühlenberg 1, D-14476 Golm, Germany; lwen@aei.mpg.de.

2 Center for Space Research, Massachusetts Institute of Technology, Cambridge, MA 02139; aml@space.mit.edu, hale@space.mit.edu.

3 Universities Space Research Association and X-Ray Astrophysics Laboratory, Code 662, NASA Goddard Space Flight Center, Greenbelt, MD 20771; corbet@gsfc.nasa.gov.
}

The known orbital periods of HMXBs range from a few hours to hundreds of days, with the majority longer than 1 day. All but one of the identified supergiant systems have nearly circular orbits with periods less than 15 days, whereas most Be star systems have eccentric orbits and periods of several tens or hundreds of days (White et al. 1995). The orbital periods are evident by means of several effects, including eclipse of the X-rays by the companion, phase-dependent absorption and scattering in a stellar wind from the companion, and absorption dips caused by nonaxisymmetric structure of an accretion disk, accretion streams, or other structures. In Be star systems, the orbital periods may be manifest as periodic X-ray outbursts that occur when the accretion rate onto the compact object is enhanced around periastron passage.

Superorbital periods have been observed in both HMXBs and LMXBs. In a handful of cases, i.e., the on-off cycles of Her X-1 (35 day period), LMC X-4 (30 days), and SMC X-1 ( $\sim 55$ days) and the 164 day jet precession cycle of SS 433, the modulation is very likely due to the presence of a precessing tilted accretion disk that periodically occults the X-ray source or guides the directions of the emitted jets (e.g., Katz 1973; Levine \& Jernigan 1982; Pringle 1996). The disk precession can be sufficiently regular to produce quasi-coherent or relatively narrow features in a Fourier spectrum made from a few years of data. In other sources, the causes of the modulation corresponding to reported superorbital periods are not well established. Indeed, the modulations may be weak and/or long compared with the duration of the observations, so the stability and significance of the effects are difficult to quantify.

Very long term quasi periodicities have been seen in several recurrent X-ray transients. The X-ray light curves in these transients are characterized by prominent outbursts separated by long periods of quiescence. The physical mechanism underlying these outbursts is still unclear. The favored scenario at present is some variation of the disk instability model (see the review by Lasota 2001). Transient behavior, as well as quasi periodicities of outburst occurrence times, is known to occur in both neutron star (NS) systems and systems comprising black hole candidates. Among them, the Rapid Burster (X1730-333), an NS system, is known to exhibit outbursts that sometimes recur every 200 days or so (but at other times the recurrence interval has been as short as $\sim 100$ days), and the black hole candidate $4 \mathrm{U} 1630-47$ is known to exhibit outbursts at 500-700 day intervals. A summary of detections of transient X-ray sources observed with the RXTE ASM before the year 2000 can be found in Bradt et al. (2000). 
Most of the known periodicities have been found in studies of one or a few sources, but there have also been a few more systematic searches for periodic signals. Priedhorsky \& Terrell (1983) analyzed data obtained between 1969 and 1976 by the Vela $5 B$ satellite on four sources in the Centaurus region and confirmed the 41.5 day period of GX 301-2, strengthened the evidence for a 187 day period in $4 \mathrm{U}$ 1145-619, and reported a period of 132.5 days in GX 304-1. Another search using Vela $5 B$ data focused on nine Galactic X-ray sources in the AquilaSerpens-Scutum region and uncovered evidence for a 199 day period in X1916-053 and a 41.6 day period in X1907+09, as well as a 122-125 day cycle in the outbursts of the recurrent transient Aq1 X-1 (Priedhorsky \& Terrell 1984b). Smale \& Lochner (1992) searched for periodicities with periods longer than 1 day in 17 confirmed or suspected LMXBs, also using data from Vela $5 B$. They confirmed the $\sim 175$ day period in X1820-303 (Priedhorsky \& Terrell 1984a) and found evidence for a $\sim 77$ day period in Cyg X-2 but reported that they did not convincingly detect the 199 day period in X1916-053; nor were long-term periods detected in 13 of the other systems. They found evidence of a 333 day period in Cyg X-3 that could also be explained by nonperiodic variability (see also Priedhorsky \& Terrell 1986). Smale \& Lochner (1992) concluded that long-term cyclic variability is rare in LMXBs. Another systematic periodicity search was done by Priedhorsky et al. (1995) on eight bright X-ray binaries detected by WATCH Eureca, but they did not find any previously unreported periodicities.

Here we report on a search for periodicities in data obtained with the All-Sky Monitor (ASM) on the Rossi X-Ray Timing Explorer $(R X T E)$. Since commencing regular observations in 1996 March, the ASM has monitored more than 400 X-ray sources. Among these, about $150 \mathrm{X}$-ray sources have been detected, with an average intensity greater than 10 mcrab for at least 1 week. We have conducted a global search for evidence of periodic behavior in 458 sources, using results from the ASM over its first $8.5 \mathrm{yr}$ of operation.

This paper is organized as follows. Section 2 describes the data and observational constraints. Section 3 describes technical details of the Lomb-Scargle (L-S) periodogram. Section 4 describes the implemented search strategies. Section 5 gives a summary of results and brief discussions of selected detections.

\section{DATA}

The ASM on board RXTE has been monitoring the sky routinely since 1996 March, and the light curves analyzed in this paper cover roughly the first 8.5 yr (up to 2004 August 26). Normally, during each orbit, up to $\sim 80 \%$ of the sky is surveyed to a depth of 20-100 mcrab, depending on the number and strength of sources in the field of view. A source is observed roughly 10 times a day.

The observations are analyzed to produce light curves for each of a set of sources listed in a specific catalog. Briefly, a set of four linear least-squares fits to the shadow patterns cast by X-rays from sources with known positions over a $90 \mathrm{~s}$ observation by one of the three Scanning Shadow Cameras (SSCs) of the ASM yields the source intensities in four energy bands (1.5-3, 3-5, $5-12$, and $1.5-12 \mathrm{keV}$ ). The intensities are usually given in units of the count rates expected if the sources were at the center of the field of view in one of the cameras (SSC 1) in 1996 March; in these units, the 1.5-12 keV Crab Nebula flux corresponds to about 75 ASM counts $\mathrm{s}^{-1}$. A detailed description of the ASM and the light curves can be found in Levine et al. (1996).

The results of the analysis are sorted by source to produce light curves for each of the four energy bands. Only those mea- surements that satisfy a set of filtering criteria are transferred to the light-curve files; the criteria include the value of the goodnessof-fit parameter (reduced $\chi^{2}$ ) and the strength of the background in the $1.5-3 \mathrm{keV}$ band. In the standard production analysis at MIT, weighted averages of the filtered results for each of the four energy bands are formed in 1 day time bins in order to reduce the effects of measurement errors on 1 day and longer timescales.

In the present analysis, we have used the source intensities estimated on the basis of individual $90 \mathrm{~s}$ observations, as well as 1 day averages derived at MIT by the RXTE ASM team. We approximately adjust the time of each 90 s observation of a given source for the projected distance of the spacecraft from the barycenter of the solar system.

For a number of reasons, the observations of a source with the ASM are obtained unevenly in time. The observation planning is done for $24 \mathrm{hr}$ time intervals, with a goal of maximizing the number of $90 \mathrm{~s}$ observations consistent with a large set of constraints. In particular, the pointing of the spacecraft, the position of the spacecraft in its orbit, the constraints imposed by the limited rotation range of the ASM drive assembly, and the slew rate of the drive assembly are among the factors that are involved in the planning procedures. Observations are only performed while the spacecraft attitude is fixed and when constraints on the aspect of the SSCs relative to the directions to the Sun and the center of the Earth are satisfied. The times of observations of most sources are correlated with orbital phase because of Earth blockage and because the detector high voltage is routinely turned off whenever the spacecraft passes through the South Atlantic Anomaly or other regions in which high fluxes of energetic particles are frequently encountered. High background levels can also be produced when a camera views the sunlit Earth; in this case, backscattered solar X-rays can be a problem when the Sun is particularly active. There are also other not-well-understood instances of high background that appear to be increasing with frequency and strength as the $R X T E$ mission proceeds. For sources near the ecliptic plane, there is an interval of typically $\sim 30$ days each year when the Sun passes nearby, as seen from Earth, in which few useful results are obtained. This is not only because the ASM has to be turned off whenever the Sun is in the field of view but also because solar X-rays scatter off internal collimator surfaces when the Sun is close to the field of view and produce high and spatially nonuniform background levels, particularly below $3 \mathrm{keV}$.

In addition to the effects that tend to yield strong components in the "window function" with periods of $\sim 96$ minutes (one spacecraft orbit), 1 day, and $1 \mathrm{yr}$, the orbital plane of the satellite undergoes retrograde precession with a period of $\sim 53$ days because of perturbations from Earth's oblateness. This naturally affects the pattern of observations of every source.

\section{ANALYSIS TECHNIQUES}

Periodicities in the ASM light curves have been sought by means of L-S periodograms. The L-S periodogram (Lomb 1976; Scargle 1982) has some advantages in the estimation of the power density spectrum of unevenly spaced data over the classic periodogram based on the fast Fourier transform (FFT). In the L-S periodogram, a maximum in the power occurs at the frequency that gives the least-squares fit of a sinusoidal wave to the data. The powers are evaluated at a set of frequencies chosen to be the same as that for the FFT, i.e., $\omega_{n}=2 \pi n / T$ for $n=$ $0,1, \ldots, N / 2$, where $T$ is the total length of the time interval covered by the data and $N$ is the number of data points (but may be set to a different value; see below). In our search, we oversample by a factor of 4 so that the frequencies are spaced by 
$\pi / 2 T$. The goal is to ensure the detection of a peak for a signal that is of borderline statistical significance and to best locate the peak. We have used the $\mathrm{C}$ codes of Press et al. (1992) to compute the periodograms. When we apply the codes to the $90 \mathrm{~s}$ time resolution data sets, $N$ is usually set to give a high-frequency limit of 30 cycles day $^{-1}$ rather than the number of data points. When we apply the codes to the 1 day intensity averages, $N$ is naturally limited to give a maximum frequency of $\frac{1}{2}$ cycle day ${ }^{-1}$.

For pure Gaussian noise, the powers calculated from the L-S periodogram are known to be exponentially distributed. In that case, the probability of detecting a power $P>P_{0}$ among a set of $N$ powers is

$$
\operatorname{Prob}\left(P>P_{0}\right)=1-\left(1-e^{-P_{0}}\right)^{N} .
$$

Two major issues must be confronted when searching for periodicities in the L-S periodograms. First, many X-ray sources show low-frequency noise in their periodograms (most significantly at $f<0.02$ cycles day $^{-1}$ ). Second, periodicities on timescales close to those noted above, i.e., $\sim 96$ minute, 1 day, $\sim 50$ day, $1 \mathrm{yr}$, and harmonics thereof, are observed in many of the periodograms. It is evident that the patterns in the window functions (i.e., the sampling intervals), together with the presence of strong signals in the sources, particularly at low frequencies, can contribute to these observed periodicities. We also believe that in many cases, the commonly seen periodicities are also due to the effects on the measured source intensities of systematic errors whose magnitudes are correlated with background levels and possibly other physical effects that are, in turn, correlated with, e.g., orbital phase, time of day, and time of year, in some complicated manner. The noise level also tends to be higher near these artificial periods. This hinders detections of intrinsic signals, as the noise is not white.

To cope with both of these types of problems, we have adopted an empirical method to search for and detect periodicities and to evaluate their significance. A periodogram can be whitened by dividing the spectrum by an estimate of the power of the underlying noise. The estimate of the underlying noise power is purely empirical; it is approximated as the local average following a method proposed by Israel \& Stella (1996). In this method, the noise power at a frequency bin $j$ is the local average of the underlying power calculated within a fixed number of frequency bins (the bandwidth) $\Delta j_{t}$. To better evaluate the shape of the red noise at low frequencies, the number of frequency bins to the right $\left(\Delta j_{R}\right)$ and to the left $\left(\Delta j_{t}-\Delta j_{R}\right)$ of bin $j$ are set equal on a $\log$ arithmic scale; i.e., $\log \left(j+\Delta j_{R}\right)-\log j=\log j-$ $\log \left[j-\left(\Delta j_{t}-\Delta j_{R}\right)\right]$. The average noise power is then calculated in the left and right frequency bands (with bins $j-2$ to $j+2$ excluded), and an average of these two numbers serves as the local average noise power $P_{\mathrm{la}}(j)$. We then compute rescaled powers $P_{r}(j)=P(j) / P_{\mathrm{la}}(j)$.

For each power spectrum, the bandwidth $\Delta j_{t}$ is chosen in such a manner that it maximizes the probability that the distribution of the rescaled powers is exponential, as expected for white Gaussian noise. To do so, we increase the bandwidth $\Delta j_{t}$ by factors of 2.5 from 30 frequency bins up to one-tenth of the total number of frequency bins in the spectrum and calculate, for each bin size, the probability that the rescaled powers follow an exponential distribution using the Kolmogorov-Smirnov test (Press et al. 1992). Once the optimal bandwidth is found, the spectrum is rescaled, and the rescaled powers are searched for significant signals. In practice, the probability that the rescaled powers follow the exponential distribution usually falls in the range $10 \%-90 \%$. Occasionally, the probability is very low $(<1 \%)$; this

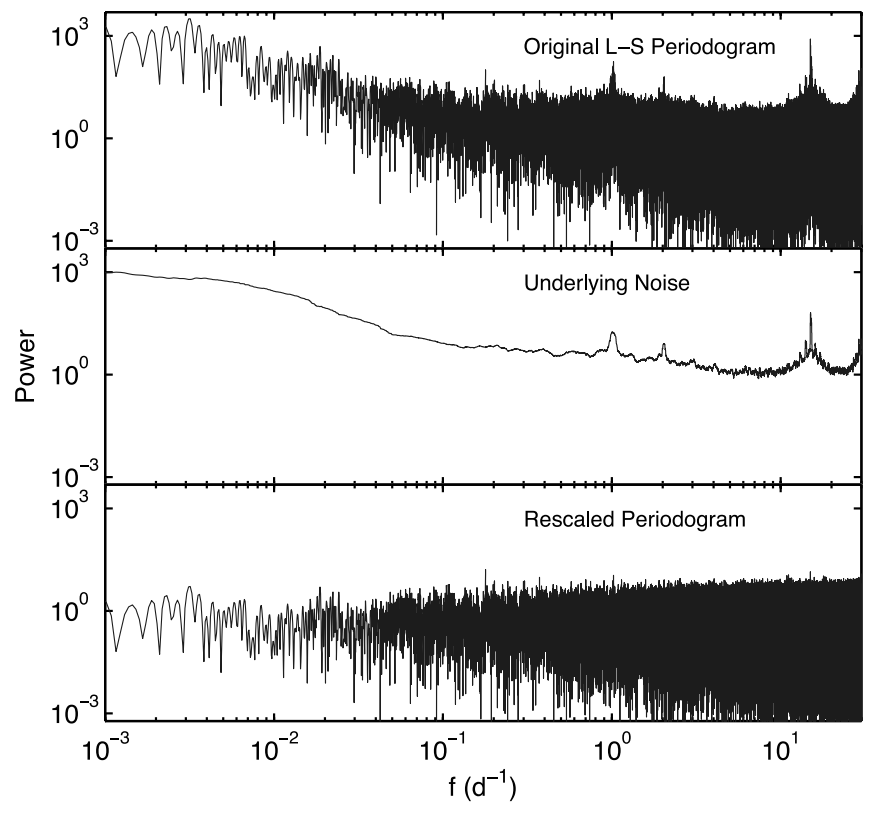

FIG. 1.-L-S periodogram of Cyg X-1, the calculated underlying noise, and the rescaled periodogram (see text). The 5.6 day orbital period of Cyg X-1 is the highest power in the rescaled periodogram and is detected at a $99.99 \%$ significance level.

may be caused by the presence of strong signals, real or artificial, their harmonics, and beats between signals. In all cases, we assume an exponential distribution for the rescaled powers.

Examples of both original and rescaled periodograms, in this case based on $8.5 \mathrm{yr}$ of data in $90 \mathrm{~s}$ time bins on the source Cyg X-1, are shown in Fig. 1. The original periodogram is shown in the top panel; the known 5.6 day orbital period is apparent. In addition, the 1 day period and its (second) harmonic, the 96 minute period, and its beat with the 1 day period are clearly present. The middle panel shows the calculated local power level, and the bottom panel shows the rescaled periodogram. The 5.6 day period is clearly detected, with the false alarm probability (FAP) of less than 0.001 . The artifacts near 1 day $^{-1}$ and harmonics are well suppressed by the rescaling method.

For each detected periodicity, we refine our estimates of the detected period and its uncertainty based on the following considerations. For a sinusoidal signal embedded in Gaussian noise, the uncertainty in the period can be calculated theoretically when the data are evenly sampled. Numerical simulations have shown that the same result holds well for unevenly sampled data (Horne \& Baliunas 1986 and references therein). Using the equation cited in Horne \& Baliunas (1986) and the relation of the L-S power to the signal-to-noise ratio in the time domain, we rewrite the uncertainty in the frequency in terms of the L-S power as

$$
\delta f=\frac{3}{8} \frac{1}{T \sqrt{P_{r}}},
$$

where $T$ is the total length of the time interval represented by the data set and $P_{r}$ is the rescaled L-S power. We have performed a simulation to confirm the validity of this estimate. In the simulation we add sinusoidal signals at frequencies and amplitudes typical of our detections to all available ASM data and then use the search procedure described above to search for periodicities within the frequency range $0<f<2 f_{\text {sim }}$ where $f_{\text {sim }}$ is the 
TABLE 1

Spin and Orbital Periodicities Detected with the $R X T E$ ASM

\begin{tabular}{|c|c|c|c|c|}
\hline Source Name & Period & FAP & $\begin{array}{c}\text { Energy Band } \\
\quad(\mathrm{keV})\end{array}$ & Comments \\
\hline \multicolumn{5}{|c|}{ NS Spin Periods } \\
\hline 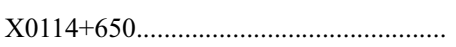 & $2.7421(2) \mathrm{hr}$ & $7 \mathrm{E}-5^{\mathrm{a}}$ & $1.5-12$ & HMXB/SG? \\
\hline X0352+30 (X Per) & $837.8(1) \mathrm{s}$ & $3 \mathrm{E}-7^{\mathrm{b}}$ & $1.5-12$ & $\mathrm{HMXB} / \mathrm{Be}$ \\
\hline \multicolumn{5}{|c|}{ Orbital Periods: CVs } \\
\hline 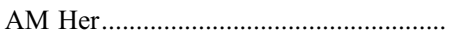 & $0.1289265(3)$ days & $1 \mathrm{E}-16$ & $1.5-12$ & Polar \\
\hline \multicolumn{5}{|c|}{ Orbital Periods: LMXBs } \\
\hline 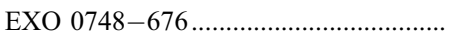 & $0.1593375(6)$ days & $6 \mathrm{E}-7$ & $3-5$ & Eclipse \\
\hline 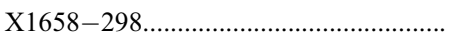 & $0.296499(4)$ days & $5 \mathrm{E}-4^{\mathrm{c}}$ & $1.5-12$ & Eclipse \\
\hline Her X-1 .......... & $1.70015(9)$ days & $5 \mathrm{E}-50$ & $5-12$ & Eclipse \\
\hline 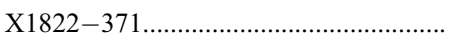 & $0.2321091(6)$ days & $3 \mathrm{E}-57$ & $1.5-12$ & $\mathrm{ADC}$ \\
\hline X2127+119 (M 15) & $0.713026(1)$ days & $3 \mathrm{E}-8^{\mathrm{c}}$ & $3-5$ & $\mathrm{ADC}$ \\
\hline 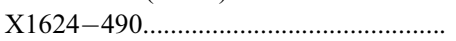 & $0.86990(2)$ days & $1 \mathrm{E}-33$ & $5-12$ & ADC, Dip \\
\hline X1916-053 & $0.03472969(5)$ days & $1 \mathrm{E}-3^{\mathrm{c}}$ & $1.5-12$ & Dip \\
\hline 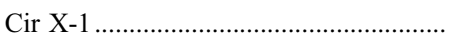 & 16.55(1) days & $5 \mathrm{E}-21$ & $1.5-3$ & Flares \& dips \\
\hline \multicolumn{5}{|c|}{ Orbital Periods: HMXB Supergiant Systems } \\
\hline 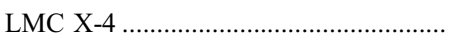 & $1.40840(6)$ days & $5 \mathrm{E}-8$ & $5-12$ & Eclipse \\
\hline 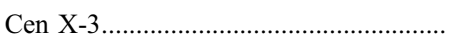 & $2.08706(9)$ days & $5 \mathrm{E}-56$ & $1.5-3$ & Eclipse \\
\hline 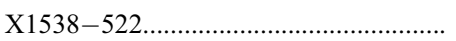 & $3.7284(3)$ days & $2 \mathrm{E}-81$ & $1.5-12$ & Eclipse \\
\hline SMC X-1 & $3.8921(4)$ days & $1 \mathrm{E}-50$ & $5-12$ & Eclipse \\
\hline 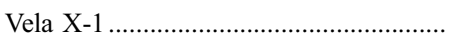 & $8.965(4)$ days & $1 \mathrm{E}-115$ & $1.5-12$ & Eclipse \\
\hline 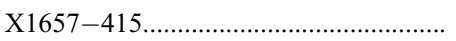 & $10.446(2)$ days & $3 \mathrm{E}-37$ & $5-12$ & Eclipse \\
\hline 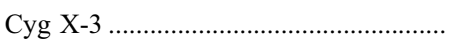 & $0.1996907(7)$ days & $1 \mathrm{E}-195$ & $5-12$ & Wind? \\
\hline 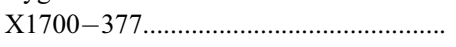 & $3.4117(2)$ days & $1 \mathrm{E}-147$ & $3-5$ & Wind \\
\hline X1908+075 & 4.4005(4) days & $7 \mathrm{E}-10$ & $1.5-12$ & Wind? \\
\hline 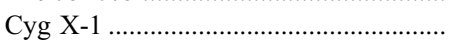 & $5.6008(7)$ days & $7 \mathrm{E}-12^{\mathrm{d}}$ & $1.5-12$ & Wind \\
\hline 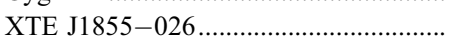 & $6.0752(8)$ days & $6 \mathrm{E}-19$ & $5-12$ & Wind? \\
\hline X1907+097 & $8.375(2)$ days & $2 \mathrm{E}-26$ & $5-12$ & Wind? \\
\hline 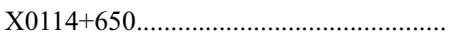 & $11.599(5)$ days & $6 \mathrm{E}-12$ & $1.5-12$ & Wind? \\
\hline 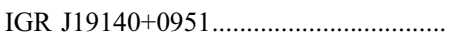 & 13.552(3) days & $1 \mathrm{E}-18$ & $5-12$ & \\
\hline IGR J00370+6122 & $15.670(4)$ days & $1 E-14$ & $1.5-12$ & \\
\hline GX 301-2 . & $41.45(6)$ days & $<4 \mathrm{E}-211$ & $5-12$ & Outburst/dip \\
\hline \multicolumn{5}{|c|}{ Orbital Periods: HMXB Be Star Systems } \\
\hline $\mathrm{X} 2206+543$ & 9.561(3) days & $6 \mathrm{E}-12$ & $1.5-12$ & Outburst \\
\hline 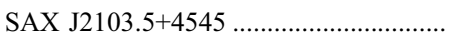 & 12.673(4) days & $2 \mathrm{E}-6$ & $1.5-12$ & \\
\hline LSI +61303 & 26.2(1) days & $4 \mathrm{E}-3^{\mathrm{e}}$ & $1.5-12$ & \\
\hline 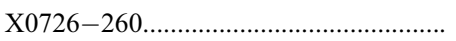 & $34.44(4)$ days & $7 \mathrm{E}-14$ & $5-12$ & Outburst \\
\hline EXO 2030+375 & 46.14(6) days & $1 \mathrm{E}-60$ & $5-12$ & Outburst \\
\hline 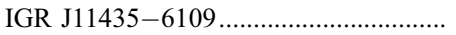 & $52.36(8)$ days & $2 \mathrm{E}-2$ & $5-12$ & \\
\hline GRO J2058+42 & $55.1(1)$ days $^{\mathrm{f}}$ & $2 \mathrm{E}-6$ & $1.5-12$ & Outburst \\
\hline RX J0812.4-3115 & $80.8(2)$ days & $4 \mathrm{E}-5^{\mathrm{d}}$ & $5-12$ & Outburst \\
\hline
\end{tabular}

${ }^{\text {a }}$ Detected in 0.4 yr (MJD 50,425.46-50,552.84).

${ }^{b}$ Detected with extended frequency range up to 120 cycles day $^{-1}$.

${ }^{c}$ FAP quoted from the first $6.5 \mathrm{yr}$ of data.

${ }^{\mathrm{d}}$ FAP quoted from the first $8.6 \mathrm{yr}$ of 1 day average data.

${ }^{\mathrm{e}}$ Detected from the first $2.5 \mathrm{yr}$ of data.

${ }^{\mathrm{f}}$ The ASM detected half the orbital period.

frequency of the simulated signal. The oversampling factor of the periodogram is set to make the frequency bin size close to what is expected from equation (2). The measured period and its deviation from the true value are recorded only if the signal is detected with an FAP of less than $10 \%$. We found that in more than $90 \%$ of the cases, the deviation between a detected period and its exact value is well bounded by the frequency uncertainty given by equation (2). Large deviations occur only at low pow- ers $\left(P_{r} \lesssim 15\right)$. The threshold in our searches is set by the FAP (see $\S 4$ ) and corresponds, in terms of power, typically to $P_{r}>$ 18. Thus, the estimate given in equation (2) is a conservative upper bound for significantly detected signals.

The periods and associated uncertainties of the detected signals are therefore determined as follows. We recalculate the rescaled L-S periodogram in the frequency range $0<f<2 f_{\text {init }}$, where $f_{\text {init }}$ is our initial preliminary estimate of the frequency of 
TABLE 2

Superorbital Periodicities Detected with the $R X T E$ ASM

\begin{tabular}{|c|c|c|c|c|}
\hline Source Name & $\begin{array}{r}\text { Period } \\
\text { (days) }\end{array}$ & FAP & $\begin{array}{l}\text { Energy Band } \\
\quad(\mathrm{keV})\end{array}$ & Comments \\
\hline \multicolumn{5}{|c|}{ Periodicities } \\
\hline 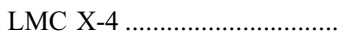 & $30.28(2)$ & $2 \mathrm{E}-91$ & $5-12$ & $\mathrm{HMXB/P}$ \\
\hline 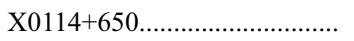 & $30.75(3)$ & $5 \mathrm{E}-9^{\mathrm{a}}$ & $5-12$ & $\mathrm{HMXB/SG?}$ \\
\hline Her X-1 ....................................... & $35.00(4)$ & $3 \mathrm{E}-20$ & $1.5-3$ & $\mathrm{LMXB} / \mathrm{P}$ \\
\hline XTE J1716-389 …...................... & $99.1(4)$ & $2 \mathrm{E}-6^{\mathrm{a}}$ & $1.5-12$ & LMXB(?) \\
\hline SS $433 \ldots \ldots \ldots \ldots \ldots \ldots \ldots \ldots$ & $162(1)$ & $3 \mathrm{E}-3$ & $5-12$ & HMXB/jet \\
\hline $\mathrm{X} 1820-303 \ldots \ldots \ldots \ldots \ldots \ldots \ldots \ldots$ & $172(1)$ & $6 \mathrm{E}-18$ & $5-12$ & LMXB \\
\hline \multicolumn{5}{|c|}{ Quasi Periodicities } \\
\hline 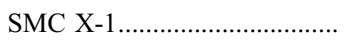 & $55.5(2)(50-70)$ & $3 \mathrm{E}-10$ & $1.5-12$ & $\mathrm{HMXB} / \mathrm{P}$ \\
\hline 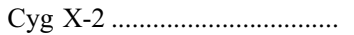 & $68.9(2)(60-90)$ & $1 \mathrm{E}-2^{\mathrm{a}}$ & $1.5-12$ & LMXB \\
\hline GRS $1747-312 \ldots \ldots \ldots \ldots \ldots \ldots \ldots$ & $146.7(9)$ & $3 \mathrm{E}-6$ & $1.5-12$ & LMXB/NS \\
\hline X1730-333...... & $217(2)$ & $2 \mathrm{E}-5^{\mathrm{a}}$ & $1.5-15$ & LMXB \\
\hline 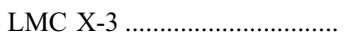 & $453(17)(100-500)$ & $2 \mathrm{E}-3^{\mathrm{a}}$ & $5-12$ & HMXB \\
\hline
\end{tabular}

${ }^{a}$ FAP quoted from the first $8.6 \mathrm{yr}$ of 1 day average data.

the detected signal. In this recalculation, we repeat the procedure as in the simulation described above; that is, we use a large oversampling factor chosen so that the frequency bin size is as close as possible to the value estimated from equation (2) while the period is still detectable at $90 \%$ confidence. Each detected period that is listed in the second column of Tables 1,2 , or 3 corresponds to the frequency of the bin that yields the maximum power in the periodogram, and the quoted uncertainty is the greater of the frequency bin size or that estimated from equation (2). For a quasi periodicity (defined as the frequency width $>1 / T$ ), we also include one-half of the frequency extent (FWHM) of the (often multiple) signal powers.

\section{DETECTION STRATEGY AND IDENTIFICATION OF ARTIFACTS}

For each periodogram, the preliminary threshold to select candidate signals was chosen to give an FAP of $<10 \%$ in the entire periodogram.

We first reject any detections that we believe to be attributable to causes other than behavior of the cosmic source. The few periodicities related to artifacts discussed previously are evident in many sources. The 1 day period has been detected in 50 sources in the frequency range $f=1 \pm 0.1$ day $^{-1}$, the 96 minute orbital period of the satellite is detected in 73 sources at $f=15 \pm 0.1$ day $^{-1}$, and the second harmonic thereof is detected in 14 sources at $f=30 \pm 0.1$ day $^{-1}$ (see also Farrell et al. 2005). The beat between these two periods at a frequency of 14 cycles day $^{-1}$ is de- tected in 15 sources; this is expected because the orbital motion of the satellite is in the same sense as the rotation of Earth. Periodicities related to the motion of Earth around the Sun have been detected in 28 sources at $f=0.0028 \pm 0.0005$ day $^{-1}$ (365 day period) and in 17 sources at $f=0.0056 \pm 0.0005$ day $^{-1}$ (187 day period). We therefore normally exclude any detections in these frequency ranges. We further exclude the frequency range $0.019 \pm 0.004$ cycles day $^{-1}$ (detected in 23 sources), which is likely linked to the 53 day precession period of the satellite orbit. These frequency ranges have been chosen empirically. We further exclude periods longer than one-fourth of the duration of the ASM light curve, as the noise tends to be strong at these low frequencies and the rescaling method does not work well in that range. We do not reject detections in these frequency ranges if we know of an independent report of the detection of a similar period based on data from other instruments.

Low-frequency detections from the sources GX 13+1, GX 1+4, GX 17+2, GX 3+1, GX 339-4, GX 354-0, GX 9+1, GX 9+9, and GX 5-1 are excluded, as each of them has large peaks (after our whitening effort) randomly distributed throughout a broad frequency range corresponding to periods of a few to several hundreds of days (but see Dieters et al. 2005; Corbet 2003).

We select detections if the FAP of detecting the signal of a given power in the entire periodogram of a source is $(1) \leq 5 \times$ $10^{-5}$ or (2) less than $10^{-1}$ and we are aware of an earlier report of variability at the same period within measurement uncertainties based on independent means, such as in other wavelengths or

TABLE 3

Periodicities Detected with the $R X T E$ ASM: Special Cases

\begin{tabular}{|c|c|c|c|c|}
\hline Source Name & $\begin{array}{l}\text { Period } \\
\text { (days) }\end{array}$ & FAP & $\begin{array}{c}\text { Energy Band } \\
\quad(\mathrm{keV})\end{array}$ & Comments \\
\hline IRAS $04575-7537 \ldots \ldots \ldots \ldots$ & $3.2324(3)$ & $5 \mathrm{E}-5^{\mathrm{a}}$ & $1.5-12$ & \\
\hline $\mathrm{X} 0115+634 \ldots$ & 24 & $\ldots^{\mathrm{b}}$ & $3-5$ & $\mathrm{HMXB} / \mathrm{Be}$, Outburst \\
\hline 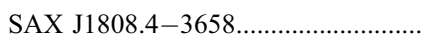 & $72.2(5)$ & $5 \mathrm{E}-5^{\mathrm{a}}$ & $3-5$ & $\mathrm{LMXB} / \mathrm{P}$ \\
\hline $\mathrm{X} 1942+274$ & 78.7 & $\ldots{ }^{\mathrm{b}}$ & $5-12$ & $\mathrm{HMXB} / \mathrm{Be}$, Outburst \\
\hline $\mathrm{X} 1145-619 \ldots \ldots \ldots$ & 185 &.$^{b}$ & $5-12$ & Outburst \\
\hline
\end{tabular}

\footnotetext{
${ }^{a}$ FAP quoted from the first $8.6 \mathrm{yr}$ of 1 day average data.

${ }^{\mathrm{b}}$ Highest peak in the original L-S periodogram but not detected in the rescaled periodogram because of its broad peak. See discussions in $\S 5.3$.
} 
using other detectors. Criterion 1 ensures that we expect a total of less than 0.1 detections at such a significance level in our searches through all $\approx 2000$ periodograms if they are random noise independently drawn from one distribution. This has proved to be reliable in practice (see $\S 5$ ). Criterion 2 is adopted because the probability of a spurious detection is proportional to the number of frequency bins searched, and hence the spurious detection of a previously known period is a factor of $\sim 10^{4}$ less probable than a spurious detection in a blind search.

It must be noted that the whitening procedure in the search technique that we have adopted tends to reduce the sensitivity to broad features and, in particular, will reduce the sensitivity to a quasi-periodic signal in proportion to the degree that the feature's breadth exceeds that of a coherent signal. This is due to the fact that the whitening procedure tends to obliterate slowly varying periodogram components, as is clearly shown in Figure 1. Thus, our search is most sensitive to coherent signals.

\section{RESULTS}

The periodicities that satisfy the criteria described above are listed in Table 1 (pulse periods and orbital periods) and in Table 2 (superorbital periods). We also list in Table 3 periodicities in five sources that require further investigations. For each periodicity, the tables give the source name, period and uncertainty therein as determined from the ASM data, and the FAP for a blind search through the $8.5 \mathrm{yr}$ (1996 March-2004 August) of data. For several sources, only the significance of a detection from a data set of shorter temporal coverage is listed (see footnotes to the tables). For these sources, the significance of the detection is lower when a data set of longer coverage is used, possibly because of a slow deterioration of the ASM data quality or possibly because the strength of the signals has decreased. We also note the nature of the systems and/or mechanisms that have been proposed for the modulation. The entries are grouped according to the possible nature of the systems, the modulation mechanisms, and the magnitudes of the periods.

We have listed a total of 51 significantly detected periodicities. Two new periodicities not published before this work, those of IRAS 04575-7537 (3.2 days) and SAX J1808.4-3658 (72.2 days), and four not previously reported in refereed publications, i.e., those in IGR J19140+098 (13.55 days), IGR J00370+6122 (15.67 days), IGR J11435-6109 (52.4 days), and XTE J1716389 (99 days), are included in the list of detections. However, the detections of the new periodicities in IRAS $04575-7537$ and SAX J1808.4-3658 may be manifestations of red noise rather than true periodicities (see $\S 5.3$ ). The tables list detections of five previously known X-ray periods that, to our knowledge, have not previously been reported to be apparent in ASM data. They are the orbital periods of the LMXBs EXO 0748-676 (3.84 hr), X1658298 (7.11 hr), X1822-371 (5.57 hr), and X1916-053 (3000.7 s) and a superorbital period in GRS 1747-312 (147 days). Our results also confirm the periodicities previously reported on the basis of X-ray intensity variations in (often much less) ASM data in X0114+650 (2.74 hr, 11.599 days, 30.8 days), X1908+075 (4.4 days), XTE J1855-026 (6.1 days), LSI+61303 (26.2 days), GRO J2058+42 (55.1 days), X2206+543 (9.6 days), X0726-260 (34.4 days), RX J0812.4-3115 (81 days), IGR J11435-6109 (52.4 days), IGR J00370+6122 (15.67 days), and IGR J19140+ 098 (13.55 days). Among them, the 30.8 day periodicity in X0114+650 was not apparent in data sets with length shorter than $6.5 \mathrm{yr}$. We did not find significant detections of a 99 or 189 day period in LMC X-3, a 42 day period in X1907+097, or a 200 day period in X1916-053 (cf. Cowley et al. 1991; Priedhorsky \& Terrell 1984b). Brief discussions of all the sources mentioned

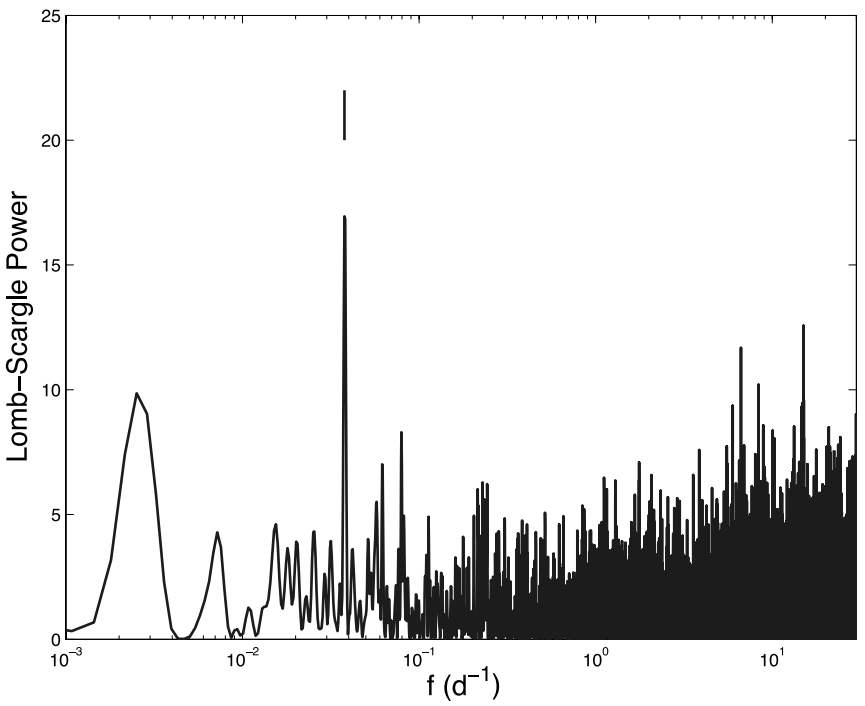

FIG. 2.-L-S periodogram of the first $2.5 \mathrm{yr}$ of $90 \mathrm{~s}$ time resolution ASM data on LSI +61303 . The 26.48 day period is indicated with a short vertical line.

above, with references to previous work, are given in $\S \S 5.1$ and 5.2, except for X1822-371 (Mason et al. 1980; White \& Swank 1982), LSI +61303 (Fig. 2; Paredes et al. 1997), X1907+097 (Baykal et al. 2001; Cox et al. 2005 and references therein), and X2127+119 (Ilovaisky et al. 1993).

With observations spanning more than $8 \mathrm{yr}$, the ASM data sometimes yield a measurement of a period that is comparable to or more accurate than previous measurements. These include the orbital periods of X1916-053, X1624-490, X0114+650, SAX $\mathrm{J} 2103.5+4545$ (see discussions in $\S 5.1$ ), and X2127+119 $\left(P_{\text {orb }}=\right.$ $0.713014 \pm 0.000001$ days; Ilovaisky et al. 1993).

We offer brief discussions of several individual detections in $\S 5.1$. The ASM light curves and periodograms are shown for some of the individual sources discussed. Periodograms for lowfrequency signals are often shown in the original L-S periodograms to show possible features around the signals. Rescaled periodograms are shown for detections at higher frequencies.

For each periodicity that is likely to be more or less coherent over the time interval used in the analysis for a given source, a set of folded light curves in the $1.5-3,3-5,5-12$, and $1.5-12 \mathrm{keV}$ energy bands has been made. The time interval used to make the folded light curve is generally $8.5 \mathrm{yr}$, i.e., ending in 2004 August, except for four cases, as noted in Table 1 . The times of the data were corrected to the solar system barycenter and folded according to the periods listed in Tables $1-3$, except for the light curve of GRO J2058+42, which was folded at twice the period listed in Table 1. Weights given by the inverse square of the estimated errors of the individual measurements were used to obtain the average intensity for each phase bin. The folded light curves are shown in Figures 3-7.

\subsection{Spin and Orbital Periods}

$\mathrm{X} 0114+650 .-\mathrm{X} 0114+650(2 \mathrm{~S} 0114+650)$ is associated with a B1 Ia optical counterpart (Reig et al. 1996). An orbital period of $11.591 \pm 0.003$ days was reported from optical radial velocity measurements (Crampton et al. 1985). Finley et al. (1992) reported a $2.78 \pm 0.01 \mathrm{hr}$ period for which there was evidence from X-ray observations with several satellites (ROSAT, Ginga, and EXOSAT), although the number of $2.78 \mathrm{hr}$ cycles in each observation was limited. The same period was also apparent in further ROSAT observations (Finley et al. 1994). Corbet et al. (1999a) 


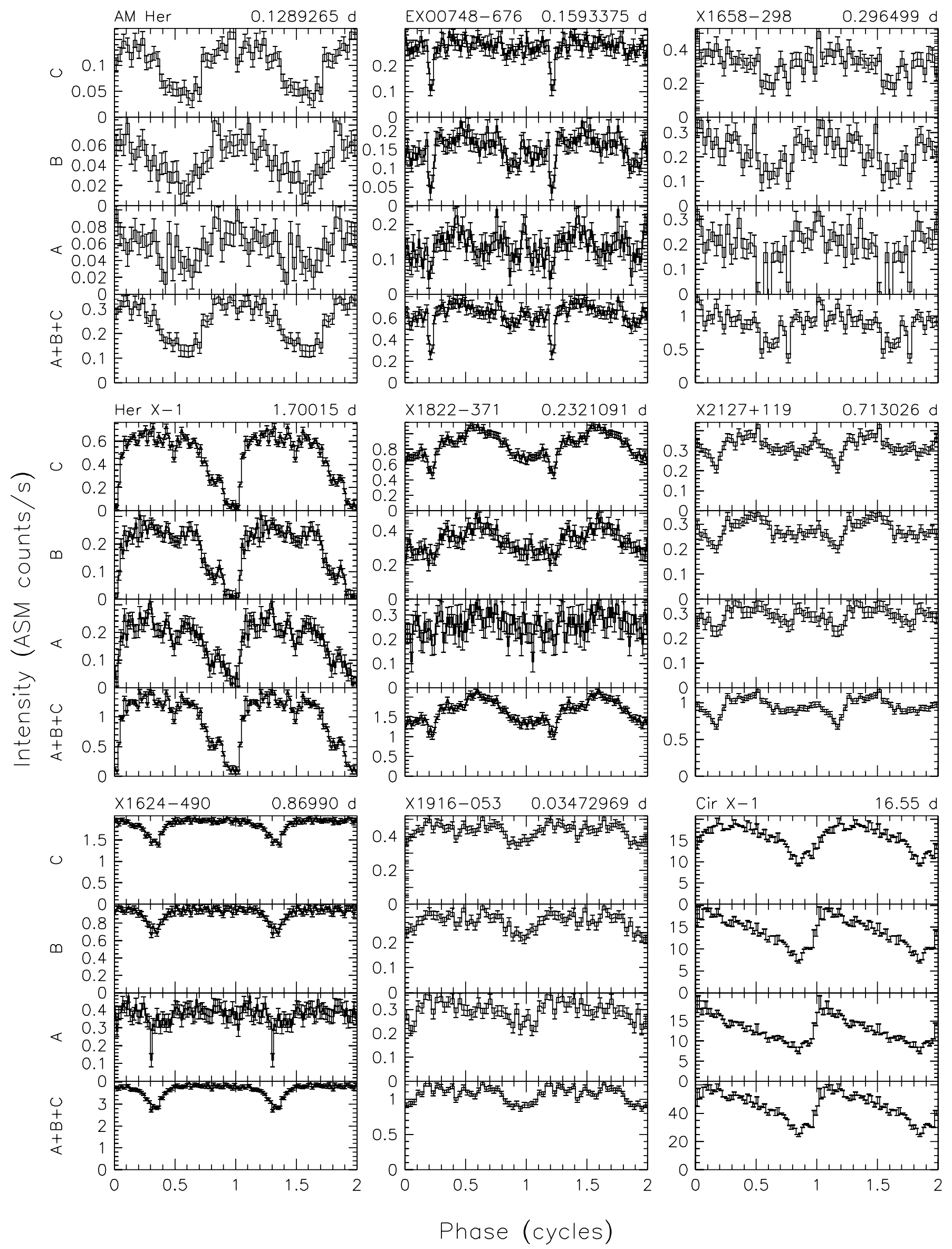

Fig. 3. - Light curves for one polar (AM Her) and eight LMXBs folded at the orbital periods from Table 1. For each source, the folded light curve is given for the $1.5-3 \mathrm{keV}(A), 3-5 \mathrm{keV}(B), 5-12 \mathrm{keV}(C)$, and $1.5-12 \mathrm{keV}(A+B+C)$ bands. For clarity, the folded light curve is shown for two full periods. 


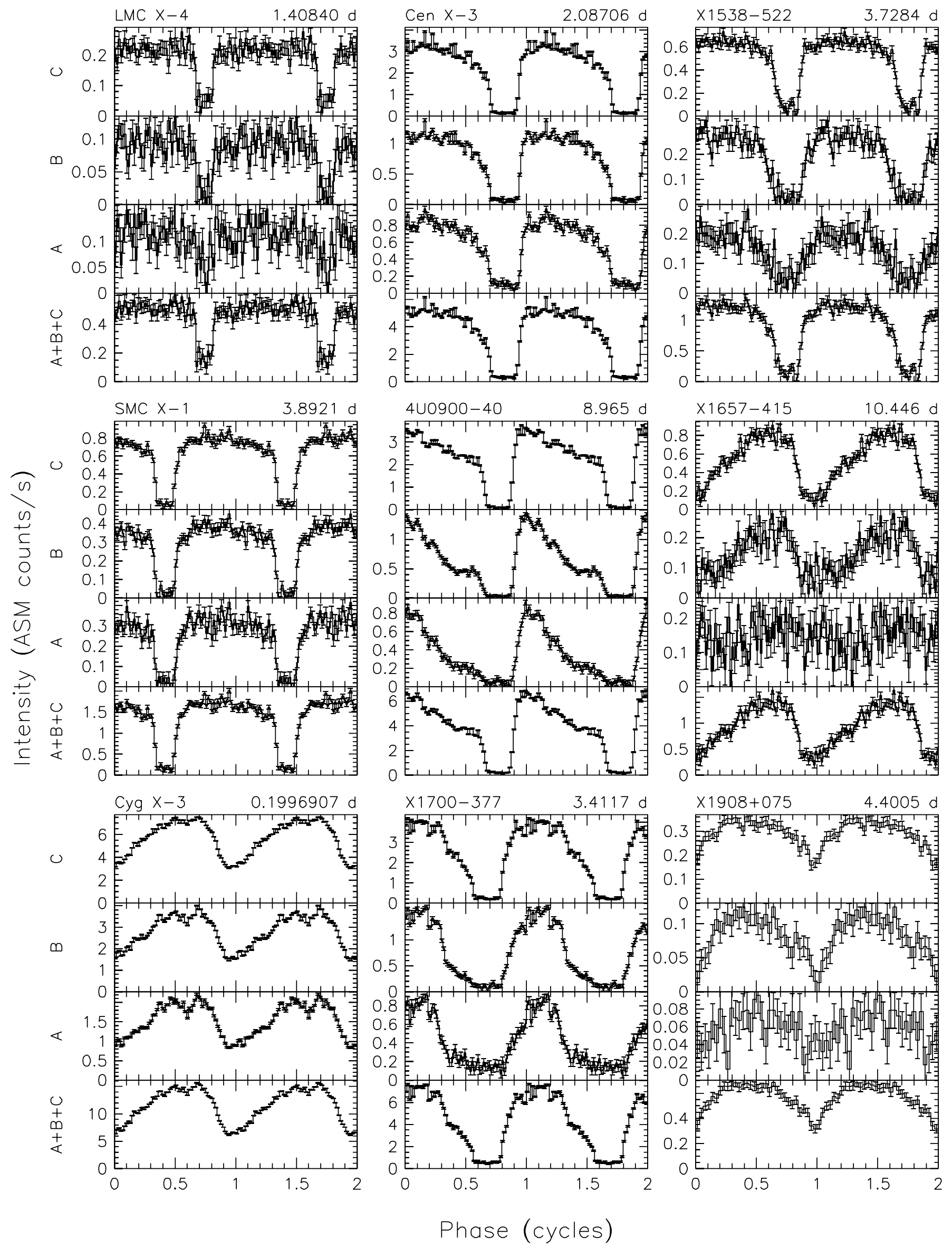

Fig. 4.-Same as Fig. 3, but for HMXB supergiant systems. 


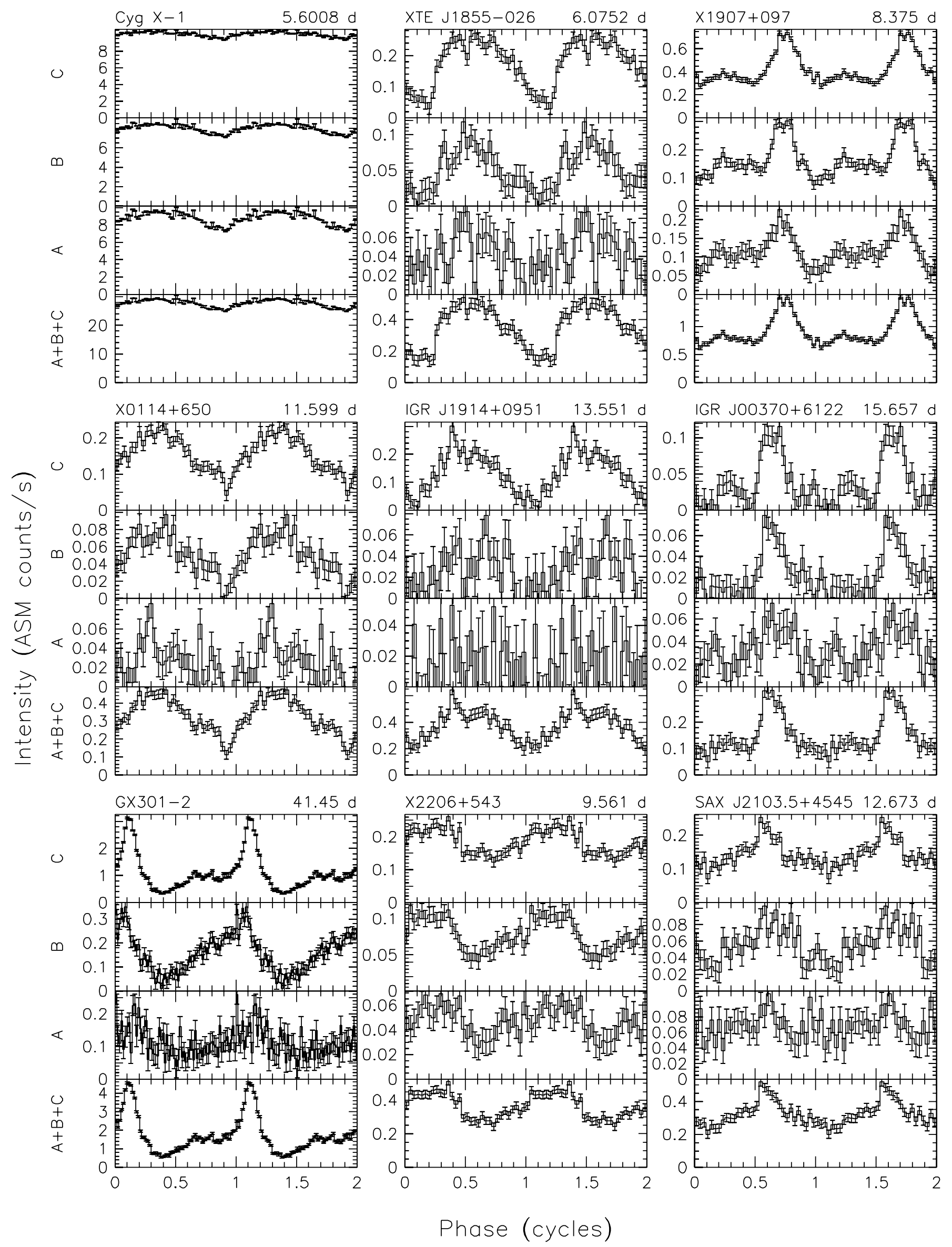

FIG. 5.- Same as Fig. 3, but for HMXB supergiant and Be star systems. 

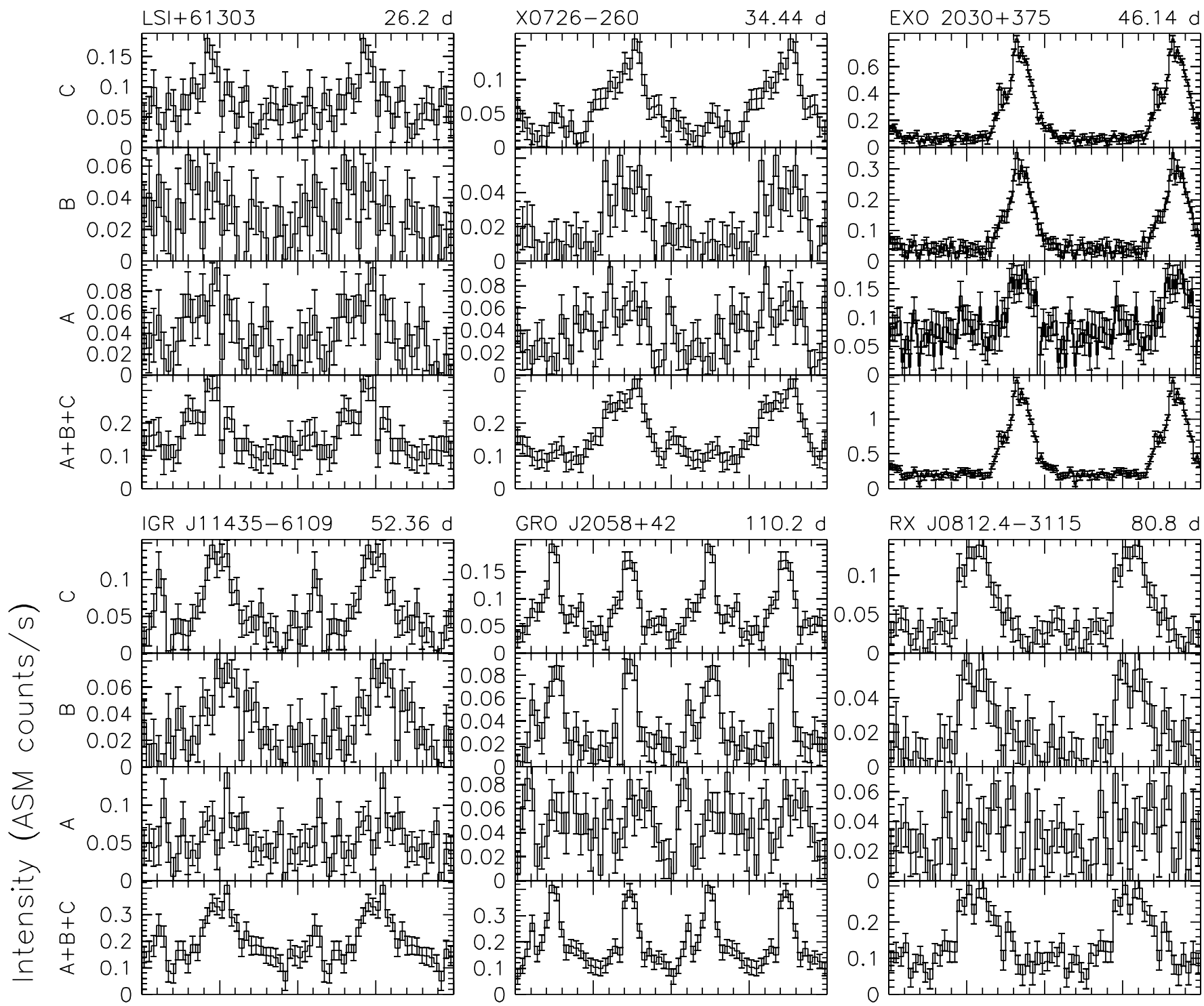


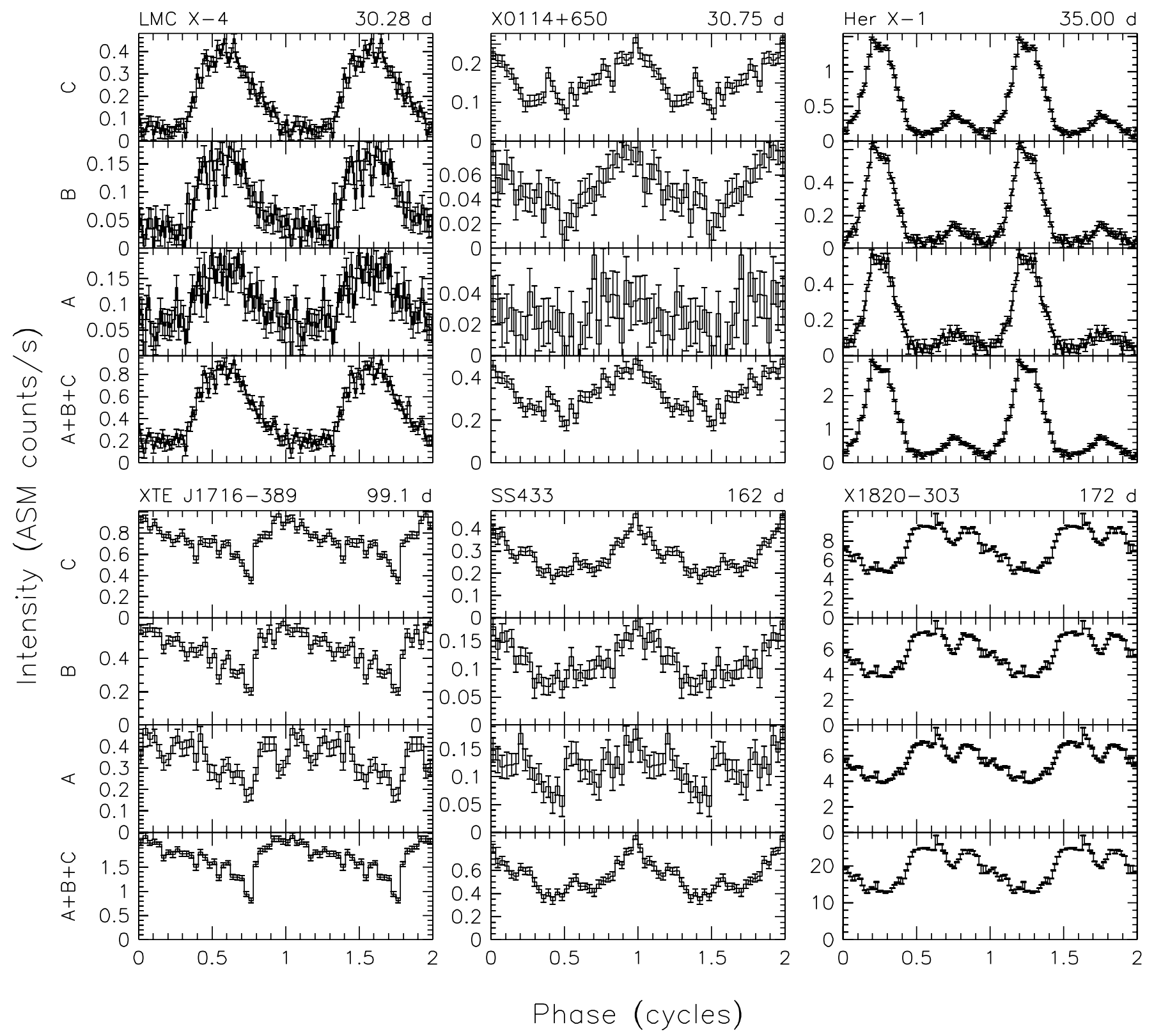

FIG. 7.-Light curves for five superorbital periodicities and one periodicity of undetermined origin (XTE J1716-389) folded at the orbital periods from Table 2 . For each source, the folded light curve is given for the $1.5-3 \mathrm{keV}(A), 3-5 \mathrm{keV}(B), 5-12 \mathrm{keV}(C)$, and $1.5-12 \mathrm{keV}(A+B+C)$ bands.

analyzed the ASM light curve of X0114+650 constructed from $2.5 \mathrm{yr}$ of observations and found modulations at both the optically derived orbital period and periods close to $2.74 \mathrm{hr}$. Their analysis of subintervals of data showed changes in the latter period that were consistent with those expected from accretion onto an NS. Thus, Corbet et al. (1999a) concluded that the $\sim 2.7 \mathrm{hr}$ period may represent the longest known NS spin period.

Using the $8.5 \mathrm{yr}$ of ASM data on X0114+650, we have significantly detected the 11.6 day orbital period and a 31 day period (Fig. 8). The $\sim 2.74 \mathrm{hr}$ (presumable) spin period can be detected significantly in a blind search using only less than $4.5 \mathrm{yr}$ of ASM data. We show in time-frequency diagrams (Fig. 9) how the frequency corresponding to the $2.74 \mathrm{hr}$ period feature appears roughly every $0.5 \mathrm{yr}$ since 1996 March. The evolution of the $\sim 2.74 \mathrm{hr}$ period is apparent. If this is indeed the NS spin frequency, the star must undergo dramatic spin changes on timescales of less than $\sim 0.5$ yr.
Detection of the 31 day period has been reported recently by Farrell et al. (2004). The origin of this periodicity is unclear. The 31 day period is not detectable using only the first $6.5 \mathrm{yr}$ of the ASM results on this source; it is most significant at the last halfyear, when the otherwise prominent 11 day period becomes less significant.

X0352+30.-X0352+30 (X Per) has been classified as a Be/ NS system. The NS shows pulsations at a period of $\sim 837 \mathrm{~s}$ (White et al. 1976). An orbital period of 250 days was revealed in a pulse timing analysis (Delgado-Martí et al. 2001). The pulsation period cannot be detected by simply applying our whitening technique to the ASM data because there are variations in the pulse period due to accretion torques that spread the power from the pulsations into many frequency bins. However, a signal appears in the unrescaled periodogram at a period of $837.8 \mathrm{~s}$ (Fig. 10). It is significant, given that the period was previously known. We found no evidence of the 250 day orbital period in the ASM data. 


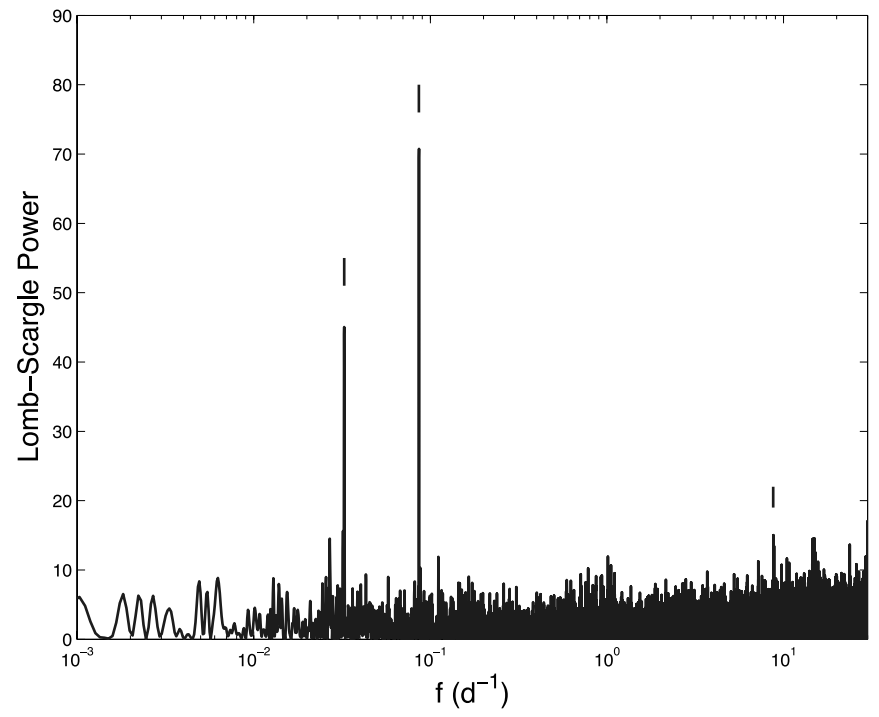

FIG. 8. - L-S periodogram from $8.5 \mathrm{yr}$ of $90 \mathrm{~s}$ time resolution ASM data in the $1.5-12 \mathrm{keV}$ band on X0114+650. The 30.8 day, 11.6 day, and $2.74 \mathrm{hr}$ periods are indicated with short vertical lines.

EXO 0748-676.-EXO 0748-676 is one of a few LMXBs in which full eclipses of the compact object are seen. It is also an $\mathrm{X}$-ray dipping source. The eclipse lasts about $500 \mathrm{~s}$ and reoccurs at the orbital period of $3.8 \mathrm{hr}$ (Parmar et al. 1986, 1991). Even though the eclipses are very brief and the source is faint $\left(F_{\mathrm{X}}\right.$ is typically $\sim 8 \mathrm{mcrab}$ ), the orbital period is detected at $3.8241 \mathrm{hr}$ with an FAP of $6 \times 10^{-7}$ in the 3-5 keV energy band with the $8.5 \mathrm{yr}$ ASM data set (Fig. 11). It is also detected in the $1.5-12 \mathrm{keV}$ band with lower confidence (FAP $\sim 1 \times 10^{-4}$ ). The periodic modulation appears to be highly energy-dependent, as it is not detected at or above $90 \%$ confidence in the $1.5-3$ or $5-12 \mathrm{keV}$ band.

X1658-298.-X1658-298, also known as MXB 1659-29, is one of the few known X-ray eclipsing and dipping sources. It is a soft X-ray transient source that reemerged in 1999 April after $21 \mathrm{yr}$ of quiescence. The $7.11 \mathrm{hr}$ orbital period was observed in

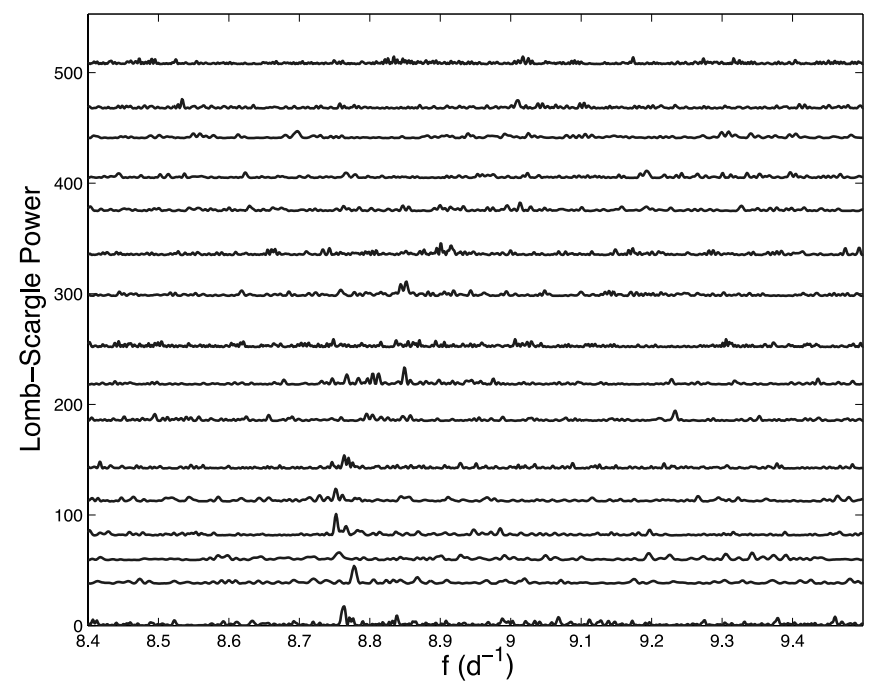

Fig. 9.-L-S periodograms ( $1.5-12 \mathrm{keV}$ band $)$ for roughly $0.5 \mathrm{yr}$ intervals of $\mathrm{X} 0114+650$. The periodograms are displaced upward, i.e., parallel to the power axis, by an amount proportional to the time of the beginning of the interval after the initial time of the first interval. The period (or frequency) changes of the signal near a period of $2.74 \mathrm{hr}$ (around 8.8 cycles day $^{-1}$ ) is apparent.

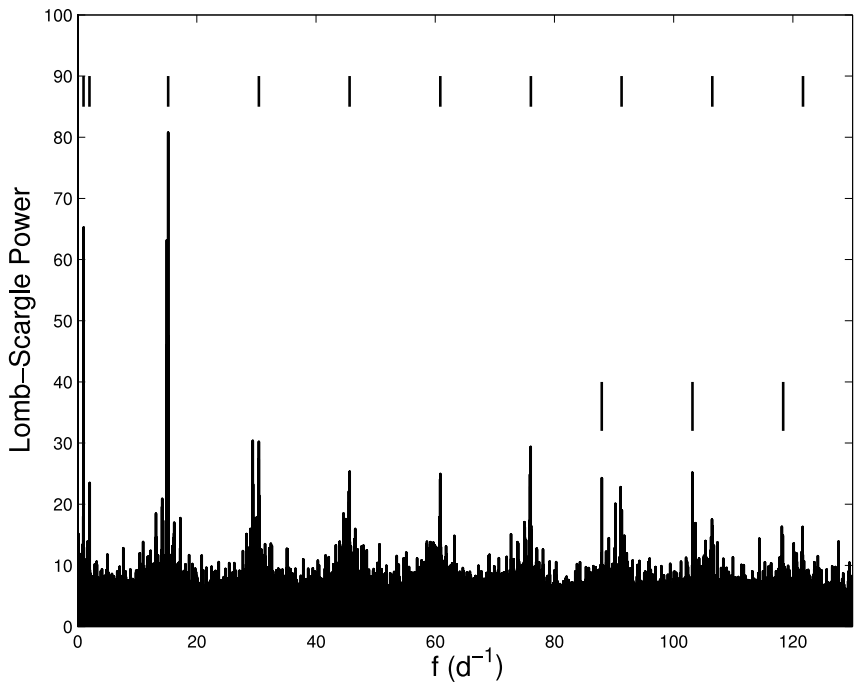

FIG. 10.-L-S periodogram of X0352+30 (X Per) in the $1.5-12 \mathrm{keV}$ band made from $8.5 \mathrm{yr}$ of $90 \mathrm{~s}$ time resolution data. The $837 \mathrm{~s}$ period $(\sim 103$ cycles day $^{-1}$ ) and its beats with the $\sim 15$ cycles day ${ }^{-1}$ orbital frequency of the spacecraft are indicated with three vertical lines. Also indicated with shorter vertical lines are the 15 cycles day $^{-1}$ spacecraft orbital frequency and seven of its harmonics, as well as the 1 cycle day $^{-1}$ rotation frequency of Earth and its second harmonic.

X-rays by Cominsky \& Wood (1989). The most recent report regarding its $7.11 \mathrm{hr}$ orbital period in X-rays after its reemergence was made by Wachter et al. (2000), using RXTE PCA data. In the present search, the period is also detected significantly in the $8.5 \mathrm{yr} 1.5-12 \mathrm{keV}$ band data set (Fig. 12). The signal is also visible in the $3-5 \mathrm{keV}$ band, with an FAP of $\sim 2 \times 10^{-2}$.

$4 U$ 1624-490.- $4 \mathrm{U} 1624-490$ is the so-called Big Dipper, one of the most unusual X-ray dipping sources. Its $21 \mathrm{hr}$ orbital period (Watson et al. 1985) is the longest among all dipping sources known. The dips can be as deep as $\sim 75 \%$ in the $1-10 \mathrm{keV}$ band. The ASM observations have provided the best data at this writing to determine its orbital period. We provide a revised period of 0.86990 (2) days, which is consistent with the value of

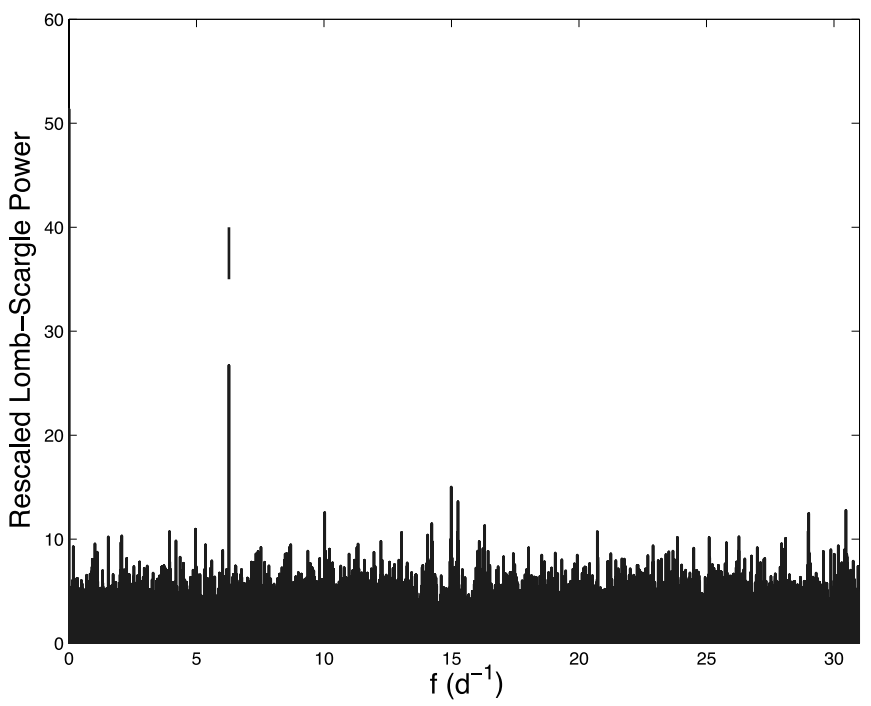

FIG. 11.-Rescaled L-S periodogram for EXO $0748-676$ in the $3-5 \mathrm{keV}$ band made from $8.5 \mathrm{yr}$ of $90 \mathrm{~s}$ time resolution data. The $3.8 \mathrm{hr}$ period is indicated with a short vertical line. 


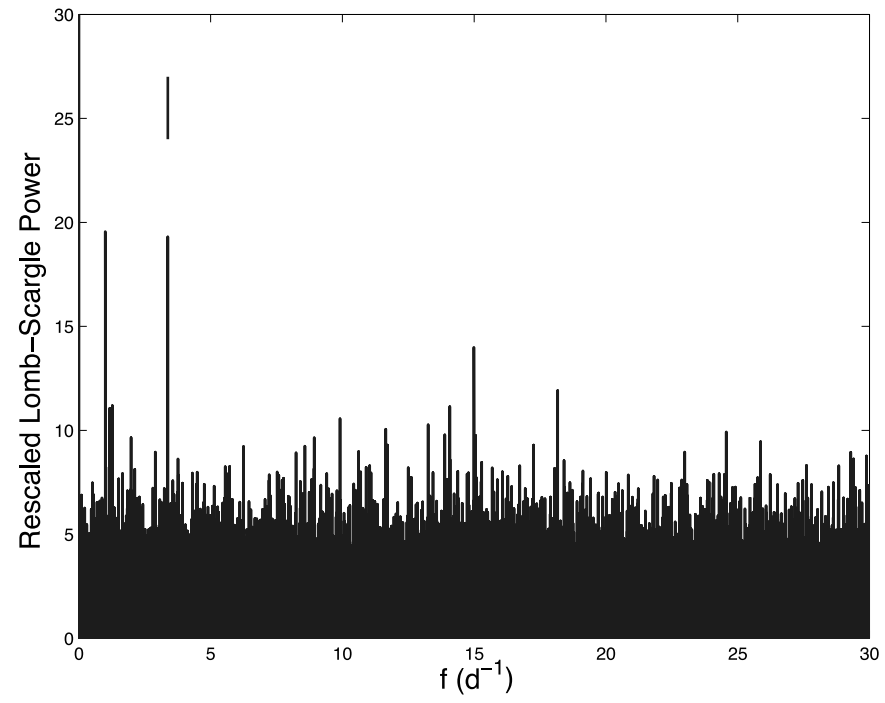

FIG. 12.-Rescaled L-S periodogram for X1658-298 in the $1.5-12 \mathrm{keV}$ band made from $6.5 \mathrm{yr}$ of $90 \mathrm{~s}$ time resolution data. The $7.11 \mathrm{hr}$ period is indicated with a vertical line. The other two high powers are artifacts at 1 and 15 cycles day ${ }^{-1}$.

$0.86991(13)$ days $(20.8778 \pm 0.003 \mathrm{hr})$ based on the analysis of $4.5 \mathrm{yr}$ of ASM data (Smale et al. 2001).

X1916-053.-X1916-053 is the most compact known X-ray dipping source. Its orbital period was reported on the basis of X-ray observations to be in the range of 2985-3015 s (Walter et al. 1982; White \& Swank 1982; Smale et al. 1989; Yoshida 1993; Church et al. 1997). A 3027 s optical period, however, is found to be stable for at least $7 \mathrm{yr}$ (Callanan et al. 1995). We have detected a coherent $3000.645 \pm 0.004 \mathrm{~s}$ orbital period, using $8.5 \mathrm{yr}$ of the ASM data (Fig. 13). This agrees with a recent report of $3000.6508 \pm 0.0009 \mathrm{~s}$ based on X-ray data from the $R X T E$ PCA, in combination with data from the Einstein, EXOSAT, and Ginga satellites (Chou et al. 2001). It was also reported in the same paper that the X-ray orbital period is not consistent with the $3027.5510 \pm 0.0052 \mathrm{~s}$ period determined from optical observa-

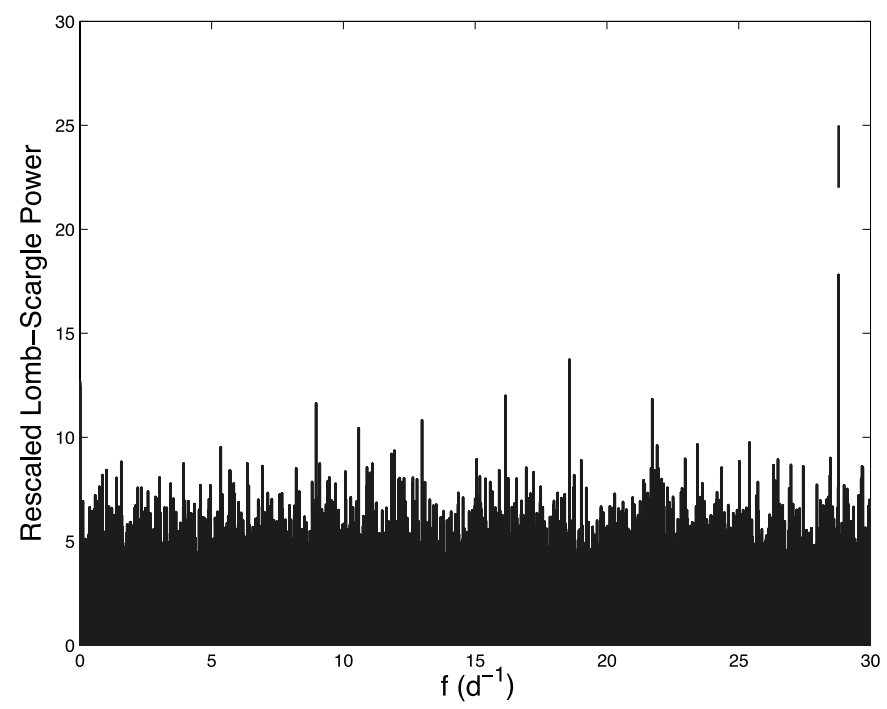

FIG. 13.- Rescaled L-S periodogram for X1916-053 in the $1.5-12 \mathrm{keV}$ band made from $6.5 \mathrm{yr}$ of $90 \mathrm{~s}$ time resolution data. The $3000 \mathrm{~s}$ period, indicated with a short vertical line, is the highest peak in the periodogram. tions. A hierarchical triple system was proposed to explain the discrepancy.

X1908+075. - X1908+075 is an HMXB with a 4.4 day orbital period that was found using $\sim 3$ yr of ASM data (Wen et al. 2000). Observations with the RXTE PCA resulted in the detection of a $605 \mathrm{~s}$ pulse period and orbital Doppler delays (Levine et al. 2004). Orbital phase-dependent low-energy absorption led to estimates of the system inclination and to the mass of the companion star. Morel \& Grosdidier (2005) have reported a likely infrared companion that could be an $\mathrm{OB}$ supergiant.

Cyg X-1.- Cyg X-1 is an HMXB that is believed to comprise an accreting $\sim 10 M_{\odot}$ black hole. Two physically distinct states of the X-ray source have been observed, the hard state and the soft state. Most of the time, Cyg X-1 stays in the hard state, where its $2-10 \mathrm{keV}$ luminosity is low and the energy spectrum is hard. Every few years, Cyg X-1 undergoes a transition to the soft state and stays there for weeks to months before returning to the hard state. During the transition to the soft state, the $2-10 \mathrm{keV}$ luminosity increases, often by a factor of more than 4 , and the energy spectrum becomes softer (see reviews by Oda 1977 and Liang $\&$ Nolan 1984 and references therein). The ASM data reveal the 5.6 day orbital period in the low state but not in the high state (Wen et al. 1999). The significance level given in Table 1 is based on an analysis of the 1 day averages including both the high- and the low-state data (see Fig. 1).

XTE J1855-026.-XTE J1855-026 was serendipitously discovered with the RXTE PCA detector during slews along the Galactic plane (Corbet et al. 1999b). The source exhibited pulsations at a period of $361 \mathrm{~s}$, and the ASM light curve revealed a period of $6.067 \pm 0.004$ days, which was interpreted as the orbital period of the system. A subsequent extended PCA light curve covering an entire 6.067 day period enabled a pulse-timing orbit to be obtained that gave a mass function of $\sim 16 M_{\odot}$ (Corbet $\&$ Mukai 2002). This mass function, together with the detection of an extended near-total eclipse, showed that the system was very likely a binary with an NS accreting from the wind of an earlytype supergiant. An improved position was obtained by the same authors using $A S C A$ data. This refined position improved the quality of the ASM light curve and, together with the use of the full $8.5 \mathrm{yr}$ of ASM data, yielded an orbital period estimate of $6.0752 \pm 0.0008$ days.

IGR J19140+098.-IGR J19140+098 (IGR J19140+0951) was discovered with INTEGRAL (Hannikainen et al. 2004). A brief observation with the $R X T E$ PCA did not detect any pulsations (Swank \& Markwardt 2003). Corbet et al. (2004) reported the discovery of a 13.55 day periodicity in the ASM light curve of this source, which they interpreted as the orbital period of an X-ray binary. Corbet et al. (2004) noted that this periodicity was present in just the first 3 yr of ASM data, and so the source was not a recent transient.

$I G R$ J00370+6122.-IGR J00370+6122 (RX J0037.2+6121) was discovered in a long INTEGRAL observation of the Cassiopeia region by den Hartog et al. (2004). A $15.665 \pm 0.006$ day period was also found in the ASM light curve, and a B supergiant counterpart was identified by den Hartog et al. The INTEGRAL error box contains the ROSAT source 1RXS J003709.6+612131. No pulsations have yet been found. Reig et al. (2005) suggest that IGR J00370+6122 shares some characteristics with the extreme LMC transient A0538-66.

X2206+543.-X2206+543 (4U 2206+54) was identified with a Be star by Steiner et al. (1984). Corbet \& Peele (2001) investigated the ASM light curve of this source and found a 9.6 day periodicity. If this is the orbital period, as appears likely, it is relatively short for a Be/NS binary and suggests that the X-ray 


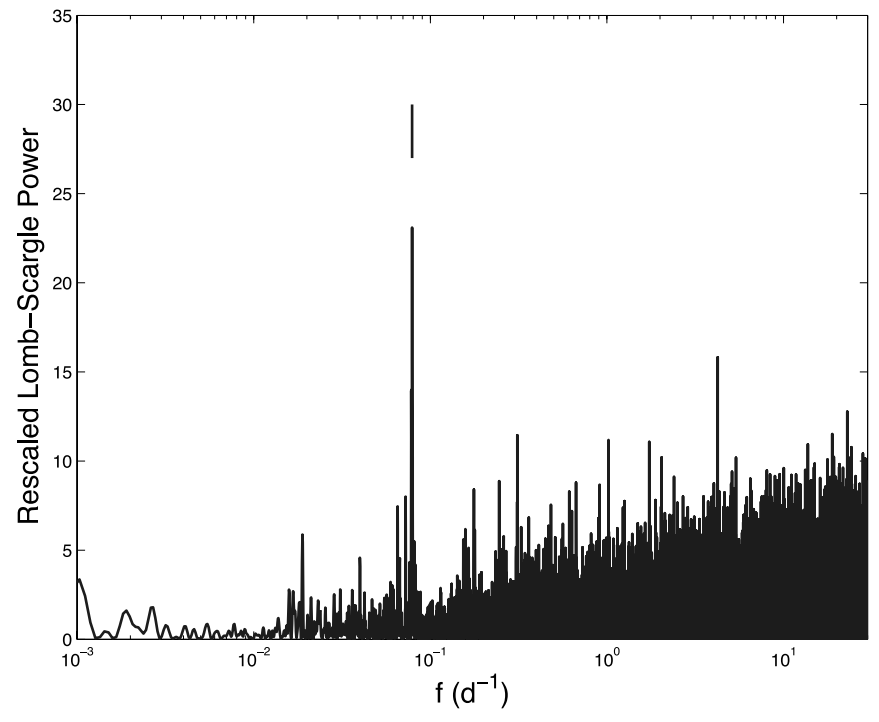

FIG. 14.-Rescaled L-S periodogram for SAX J2103.5+4545 in the 1.5$12 \mathrm{keV}$ band made from $8.5 \mathrm{yr}$ of $90 \mathrm{~s}$ time resolution data. The 12 day period is indicated with a vertical line. The second largest peak is at a $2.86 \mathrm{hr}$ period with an FAP of $\sim 0.1$.

luminosity should be high. The measured flux, however, corresponds to a luminosity of only $\sim 10^{35} \mathrm{ergs} \mathrm{s}^{-1}$, if the source is at the estimated distance of $\sim 3 \mathrm{kpc}$. PCA observations and archival EXOSAT observations have failed to reveal any evidence of pulsations (Corbet \& Peele 2001) such as were reported earlier by Saraswat \& Apparao (1992). The short orbital period and low luminosity may be at least partially reconciled by the reclassification of the optical counterpart as a peculiar O9 III-V star (Negueruela \& Reig 2001).

SAX J2103.5+4545.-SAX J2103.5+4545 is an X-ray pulsar with a $358 \mathrm{~s}$ pulse period in an orbit with a $12.68 \pm 0.25$ day period (Hulleman et al. 1998; Baykal et al. 2000). Reig et al. (2004) identified the optical counterpart as a Be star. The orbital period is easily detectable in the ASM data, and our analysis yields $P_{\text {orb }}=12.673 \pm 0.004$ days (see Fig. 14). The orbital period is one of the shortest known for a Be/NS system.

X0726-260.-X0726-260 (4U 0728-25) has an optical counterpart of spectral type O8-9Ve (Negueruela et al. 1996). From an analysis of an RXTE ASM light curve spanning $1.5 \mathrm{yr}$, Corbet \& Peele (1997) found evidence for a 34.5 day orbital period. This was apparently confirmed when a PCA observation made at the predicted time of flux maximum showed the source to be in a bright state. Corbet \& Peele (1997) also found $103.2 \mathrm{~s}$ pulsations in the X-ray flux. We have detected the 34.5 day period in the $8.5 \mathrm{yr}$ data set with improved significance.

IGR J11435-6109.-IGR J11435-6109 was reported as a hard X-ray transient by Grebenev et al. (2004). Evidence of the possible presence of $166 \mathrm{~s}$ pulsations was reported by Swank \& Markwardt (2004) and was confirmed by BeppoSAX Wide Field Camera observations by in 't Zand \& Heise (2004). In 't Zand \& Heise also reported a possible 52.5 day outburst recurrence period from detections in 1996-1997 and 2001-2002, but nondetection during the intervening period. This outburst period was confirmed by Corbet \& Remillard (2005) from RXTE ASM observations. An initial suggestion that IGR J11435-6109 corresponds to 1RXS $\mathrm{J} 114358.1-610736$ is apparently excluded by optical observations of the optical counterpart of 1RXS J114358.1-61073 by Torrejón \& Negueruela (2004), who instead propose a Be star counterpart that is 1.2 away from $2 \mathrm{E} 1141.6-6050$.

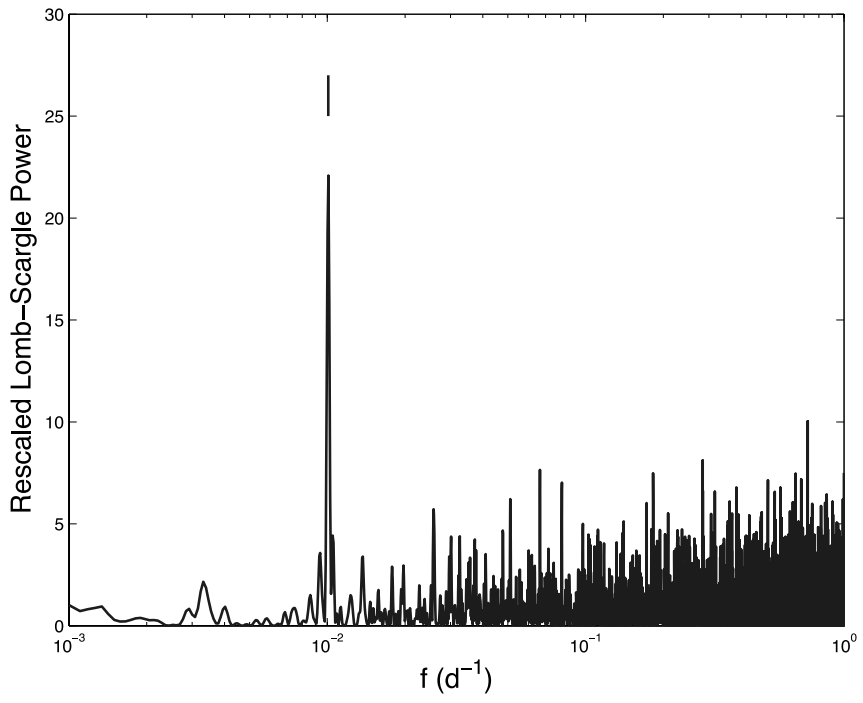

FIG. 15.-Rescaled L-S periodogram for XTE J1716-389 in the 1.5$12 \mathrm{keV}$ band made from $8.5 \mathrm{yr}$ of 1 day time averages. The $\sim 98$ day peak is indicated with a vertical line.

GRO J2058+42.-GRO J2058+42 is a transient $198 \mathrm{~s}$ X-ray pulsar also suspected to be a Be star system (Wilson et al. 1998). Both ASM and BATSE data show a number of outbursts from this source at intervals of 55 days, although a set of outbursts that appeared at intervals of 110 days were particularly strong in the BATSE data (Corbet et al. 1997; Wilson et al. 1998). It is not clear which of these intervals, if either, is the orbital period. The detection of the 55 day period is unambiguous in the ASM data, even though it nearly coincides with the precession period of the orbital plane of the $R X T E$ satellite.

$R X J 0812.4-3114$. - RX J0812.4-3114 was found to exhibit pulsations at a period of $31.9 \mathrm{~s}$ in data obtained with the $R X T E$ PCA by Reig \& Roche (1999), who concluded that it is a Be/NS binary system. As seen in the RXTE ASM light curve, it underwent a transition in early 1998 from an inactive state to a state in which short outbursts occurred every $\sim 80$ days. Corbet $\&$ Peele (2000) have interpreted the interval between outbursts as the orbital period. Our visual inspection of the ASM light curve indicates that the series of outbursts lasted for $\sim 800$ days, and our analysis confirms the detection of the $\sim 80$ day period.

\subsection{Superorbital Periods}

XTE J1716-389.-XTE J1716-389 is probably the same source as KS 1716-389, which was seen in 1994 with the TTM instrument (Aleksandrovich et al. 1995). A 99 day period is detected in the $8.5 \mathrm{yr}$ ASM data set (Fig. 15). However, our most significant detection was made using an ASM data set spanning a $4.5 \mathrm{yr}$ interval beginning in 1996 February in which a $97.51 \pm$ 0.12 day period is detected in the L-S periodogram with an FAP of less than $3 \times 10^{-7}$. The peak in the periodogram is narrow, with a width (FWHM) of 5 days, indicating detection of a highly periodic modulation. The uncertainty in the period is the estimated standard deviation, assuming a single sinusoidal signal with Gaussian noise (Horne \& Baliunas 1986). No other periodicities, excluding known artifacts, have been detected in the ASM data in the frequency range $0.001-30$ cycles day $^{-1}$.

The ASM light curve shows the source to have generally persisted at an intensity of $\sim 25$ mcrab from the beginning of operation of the ASM in 1996 February until approximately 2003 June 10 (MJD 52,800), after which the intensity is less than 10 mcrab. 


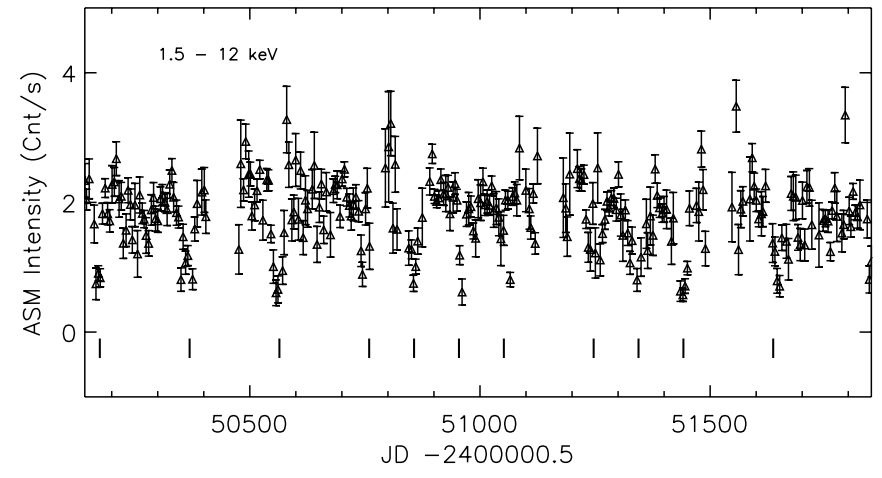

FIG. 16.-Section of the 1.5-12 keV ASM light curve of XTE J1716-389 in 5 day time bins. The visible dips are labeled with short vertical lines.

Despite its relatively low intensity, prominent dips, which may be associated with the $\sim 100$ day period, are apparent in the light curve (Fig. 16). In the dips the intensity may decrease to less than $10 \%$ of the long-term average count rate. The total duration of a dip may be as much as $\sim 40$ days. Results from both ASM and PCA observations illustrating the variation of the intensity through three dips are shown in Fig. 17. An analysis of the timing and spectral properties of the source may be found in Wen (2001). Throughout the broad dips, the PCA spectra exhibit variable but enhanced absorption. The ephemeris for the intensity minima based on the data for the interval 1996 February-2000 August is

$$
T_{n}=\operatorname{MJD} 50,174.24 \pm 0.72+n \times(97.51 \pm 0.12) .
$$

These times of minima were obtained from a fit of a sinusoid to the ASM data using the Levenberg-Marquardt (L-M) method (Press et al. 1992), which, in turn, relies on $\chi^{2}$ minimization. The uncertainty in $T_{n}$ is the estimated standard deviation from the fit.

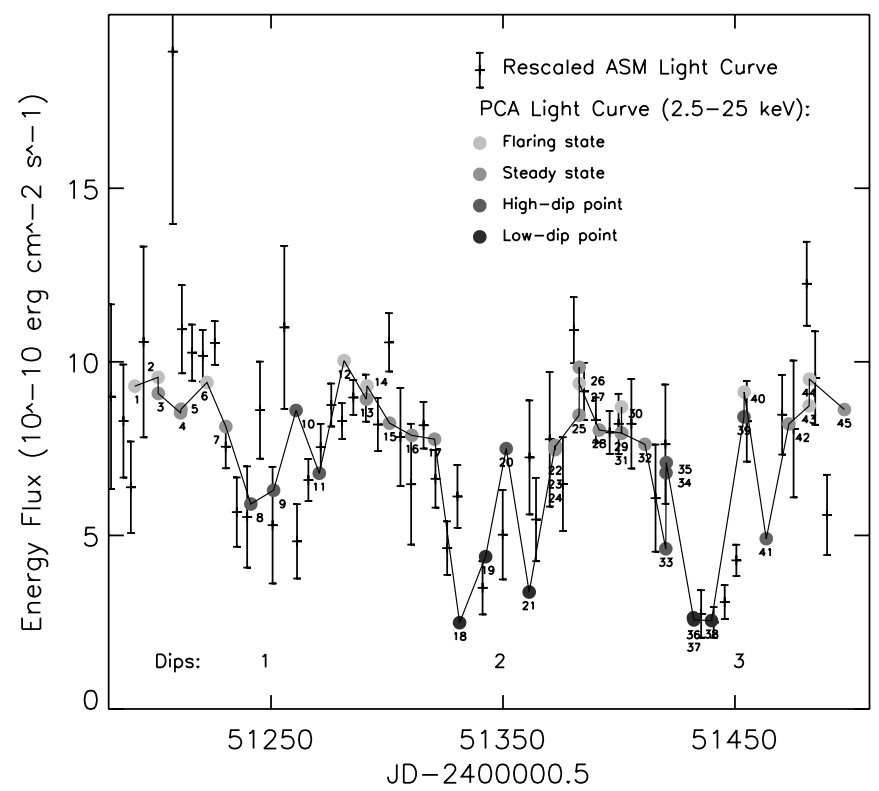

FIG. 17.-The 2.5-25 keV PCA energy flux of XTE J1716-389 as a function of time and a rescaled ASM light curve in 5 day time bins. The observation sequences are labeled. Darker shades of the filled circles indicate increasing amount of absorption column density required for spectral fitting in the PCA data. Three broad dips are apparent in both light curves. The PCA data further confirm the detection of the periodic dips in this source. [See the electronic edition of the Journal for a color version of this figure.]

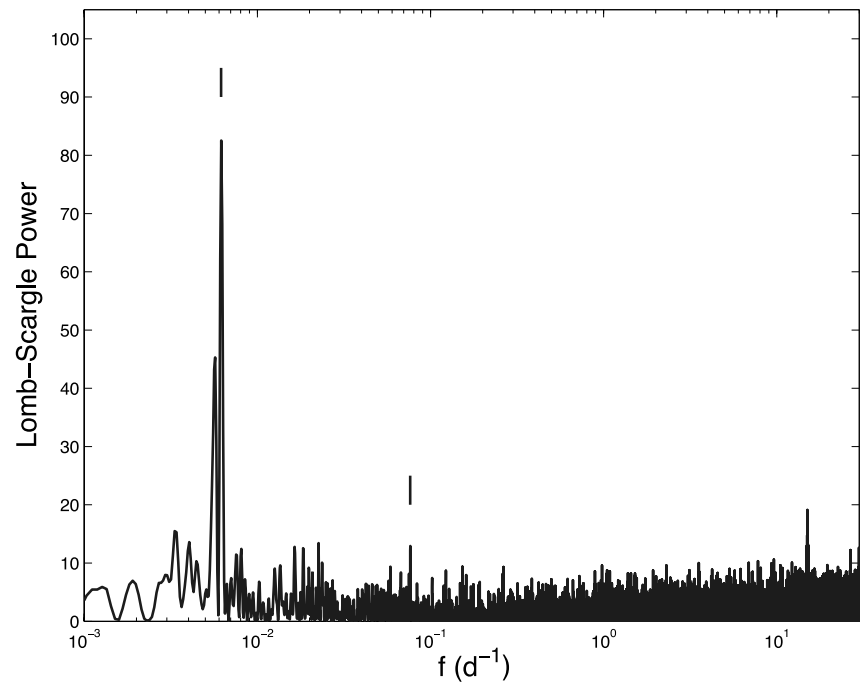

FIG. 18.-L-S periodogram for SS 433 in the $5-12 \mathrm{keV}$ band made from $8.5 \mathrm{yr}$ of $90 \mathrm{~s}$ time resolution data. The 162 day precession period and the 13.1 day orbital period are indicated with vertical lines.

If the X-ray emission from XTE J1716-389 is powered by Roche lobe overflow in a circular or nearly circular binary orbit with a 98 day period, then the mass donor star would need to be in the giant phase of its evolution (see, e.g., Verbunt \& van den Heuvel 1995). If, alternatively, the mass transfer is the result of accretion from a wind in an HMXB, the companion star would likely need to be fairly massive and evolved, so that sufficient mass to power the X-ray source would be captured by the compact object. Precession of a tilted disk like that observed in Her $\mathrm{X}-1$, LMC X-4, or SMC X-1 is perhaps a more viable model, in which case the orbital period would be substantially shorter than 98 days.

SS 433.-SS 433 is a well-known Galactic jet source. Optical emission lines that undergo dramatic motions in wavelength show that the system emits two oppositely directed jets at velocities of $\sim 0.26 \mathrm{c}$ that precess with a period of $\sim 164$ days (see, e.g., Margon 1984; Eikenberry et al. 2001). The jets are also manifest in many other ways, notably including radio emission (e.g., Blundell et al. 2001) and the Doppler shifts of X-ray emission lines (e.g., Kotani et al. 1996; Marshall et al. 2002; Migliari et al. 2002). The system is believed to be a binary comprising a massive normal-type star and an accreting compact object; thus, it may be very similar to an HMXB. The orbital period is 13.1 days (Crampton et al. 1980). The nature of the compact object is still unknown.

We have detected at a high degree of confidence a period of 162 days in the ASM data. This period is consistent with the previously established precession period (Fig. 18). A peak is also found in the periodogram at $13.090 \pm 0.001$ days, which is consistent with the orbital period. In the rescaled power spectrum, the FAP offinding such a peak at a given frequency is 0.005 . Evidence for detection of these periodicities in the ASM data has previously been presented by Gies et al. (2002).

X1820-303.-X1820-303 resides in the globular cluster NGC 6624. It was the first identified type I X-ray burster (Grindlay et al. 1976). The intensity of the source is known to vary with a period of about 176 days (Priedhorsky \& Terrell 1984a; Smale \& Lochner 1992). This periodicity is easily seen in the ASM light curve and is clearly detected in the rescaled L-S periodogram (Fig. 19). We did not detect the known $685 \mathrm{~s}$ orbital modulation (Stella et al. 1987) in an extended search. 


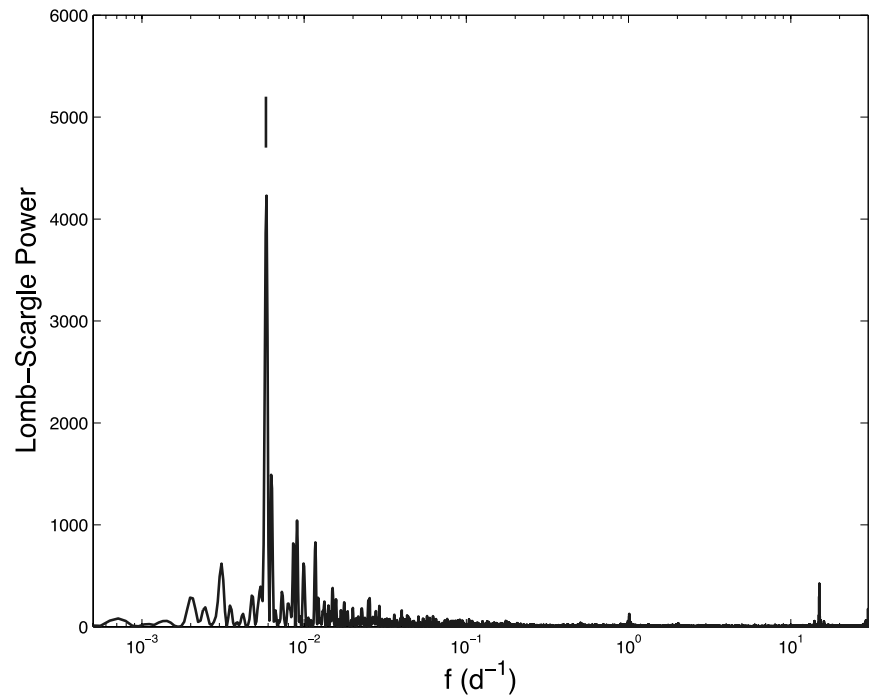

FIG. 19.-L-S periodogram for X1820-303 in the $1.5-12 \mathrm{keV}$ band made from $8.5 \mathrm{yr}$ of $90 \mathrm{~s}$ time resolution data. The 172 day period is indicated.

GRS 1747-312.- GRS $1747-312$ is a bright transient X-ray source in the globular cluster Terzan 6. It exhibits complete eclipses that recur at the orbital period of 0.52 days (in 't Zand et al. 2000, 2003). Outbursts occur approximately every 4.5 months, and type I thermonuclear X-ray bursts have been found during some of them. We have detected a 147 day period consistent with previous observations of these quasi-periodic outbursts (Fig. 20). The orbital period is not detected.

SMC X-1.-SMC X-1 is an eclipsing HMXB/NS system with a 3.89 day X-ray orbital period (Schreier et al. 1972; Levine et al. 1993). $A \sim 60$ day quasi periodicity was suggested by Gruber \& Rothschild (1984) from HEAO-1 (A-4) data and was confirmed by CGRO BATSE observations and early RXTE ASM results (Wojdowski et al. 1998; Clarkson et al. 2003). In our search, there are a number of prominent features in the periodogram corresponding to periods around $\sim 56$ days (Fig. 21). In particular, peaks that are more than $\sim 0.5$ times as large as the most promi-

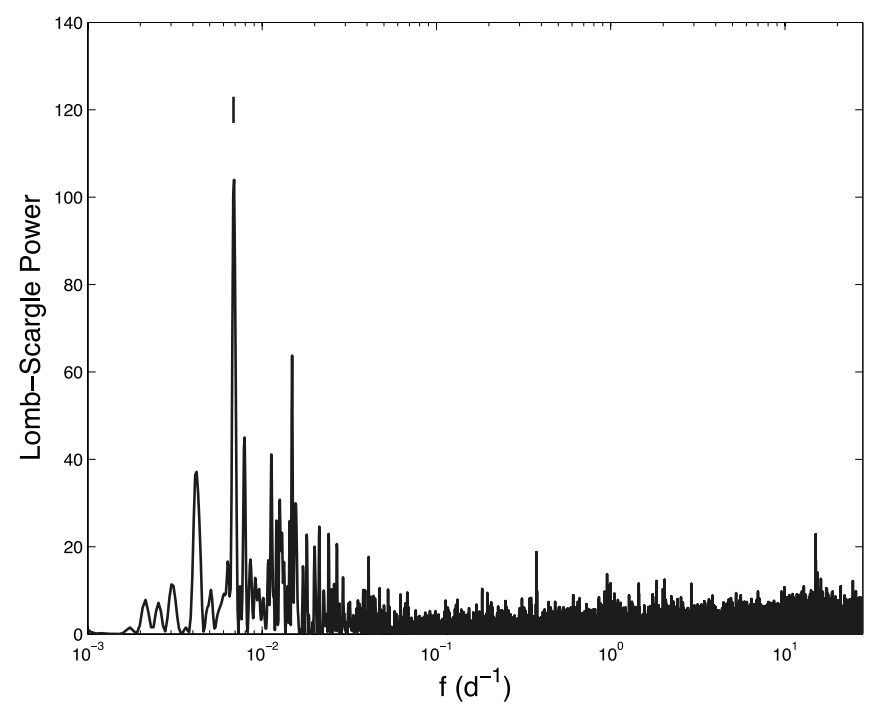

FIG. 20.-L-S periodogram for GRS $1747-312$ in the $1.5-12 \mathrm{keV}$ band in $90 \mathrm{~s}$ time bins. The quasi period of 146 days is indicated.

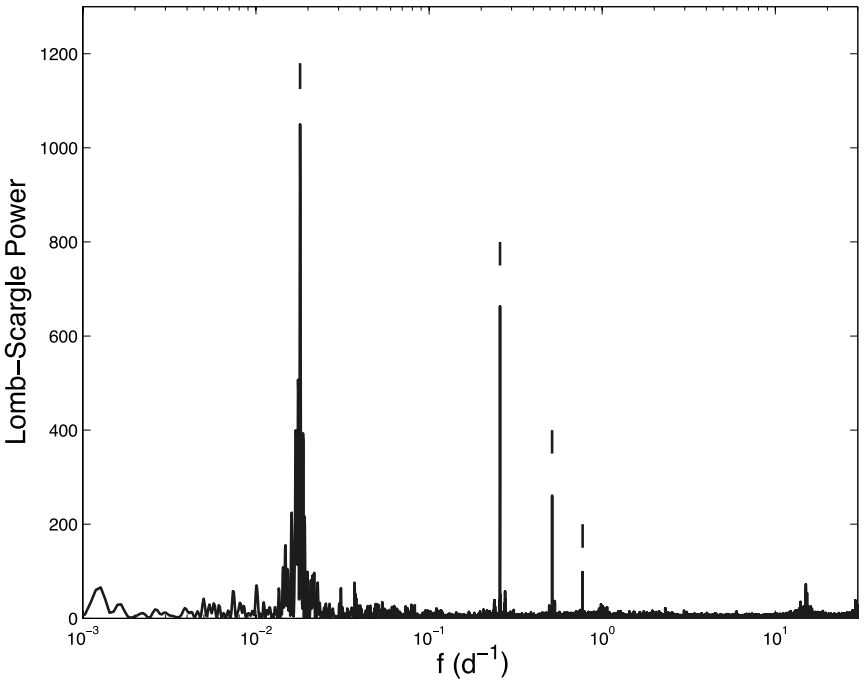

FIG. 21.-L-S periodogram for SMC X-1 in the $1.5-12 \mathrm{keV}$ band made from $8.5 \mathrm{yr}$ of $90 \mathrm{~s}$ time resolution data. The detection of the $\sim 60$ day period and the 3.9 day orbital periods and their harmonics are clear. There are multiple peaks and broad features on time scales of 50-70 days.

nent peak occur in the period range $\sim 53$ to $\sim 59$ days. The existence of closely spaced multiple peaks suggest that this is inherently a broad feature. This is consistent with previous conclusions that the $\sim 56$ day cycle is quasi-periodic. The 3.9 day orbital period and its harmonics are detected significantly. As expected, they do not show structure similar to that seen around the $\sim 56$ day feature.

Cyg $X-2$ - - Cyg X-2 is one of the brightest LMXB/NS systems. It has an orbital period of 9.8444 days (Cowley et al. 1979; Casares et al. 1998). Smale \& Lochner (1992) reported the presence of a 77 day period in the X-ray intensity. Paul et al. (2000), on the basis of the first few years of RXTE ASM data, concluded that there is no particular stable long-term period. However, Boyd \& Smale (2004) report that the long-term variations in Cyg X-2 can

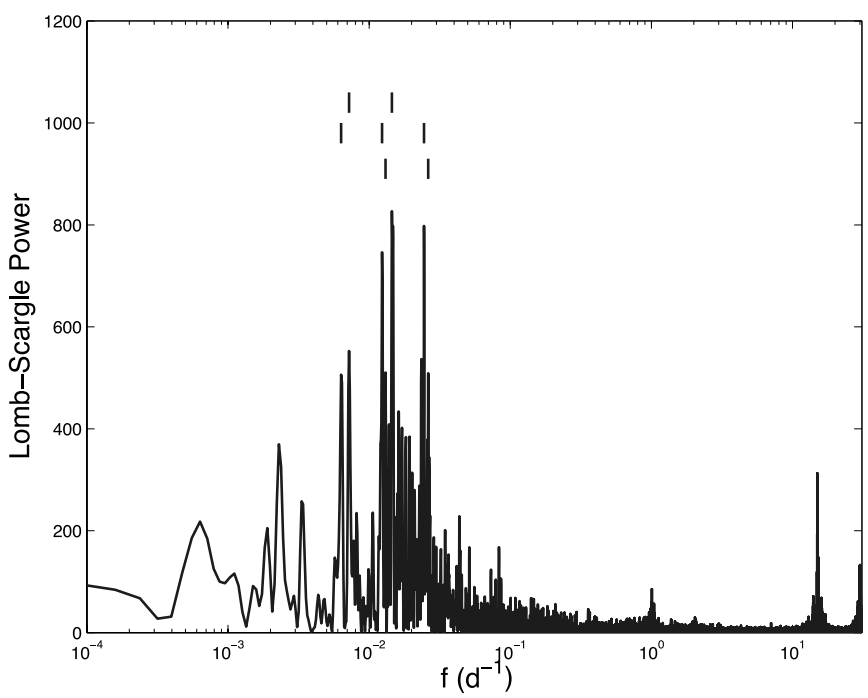

FIG. 22.-L-S periodogram for Cyg X-2 in the $1.5-12 \mathrm{keV}$ band made from $8.5 \mathrm{yr}$ of $90 \mathrm{~s}$ time resolution data. There are multiple peaks at frequencies of less than 0.03 cycles day $^{-1}$. Three possible sets of periodicities at $76.5,138.7$, and 157 days, with harmonics, are indicated with short vertical lines. The peaks at 1 and 15 cycles day $^{-1}$ are artifacts (see text). 


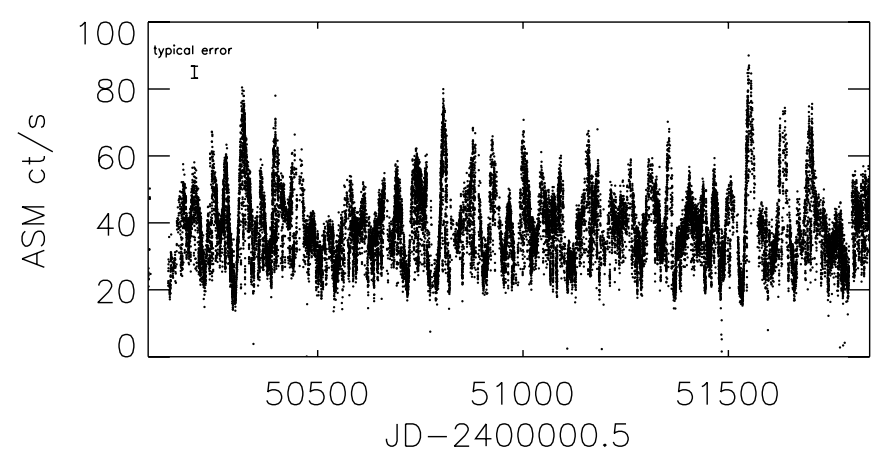

FIG. 23.-ASM 1.5-12 keV band light curve of Cyg X-2. Quasi-periodic intensity variations on timescales between 60 and 90 days are clear.

be attributed, at least in part, to about half-a-dozen periodicities with periods that are integer multiples of the orbital period. In agreement with these results, we find no single dominating periodicity in the $8.5 \mathrm{yr}$ data set. Instead, multiple peaks are apparent in the L-S periodogram (Fig. 22). Quasi-periodic intensity variations are evident in the ASM light curve (Fig. 23).

$L M C X-3$. - LMC X-3 is one of the persistent X-ray-emitting stellar-mass black hole candidates. Multiple peaks are visible in its periodogram at timescales in the range 100-500 days (Fig. 24). The variability is clearly apparent in the ASM light curves (Fig. 25). Our results are consistent with a previous report by Paul et al. (2000) that there is no stable long-term period in this source. Neither the 99 day nor the 189 day period (Cowley et al. 1991) is significant in the rescaled periodogram.

\subsection{Special Cases}

In this subsection, we discuss our results for five sources that formally indicate the presence of periodicities but should be regarded as tentative and thus in need of confirmation in independent investigations. Two types of possible periodicities that showed up in the course of our search are discussed here: (1) previously unknown periodicities detected with marginal significance by our method (3.23 days in IRAS $04575-7537$ and 72 days in SAX J1808.4-3658) and (2) previously known periodicities

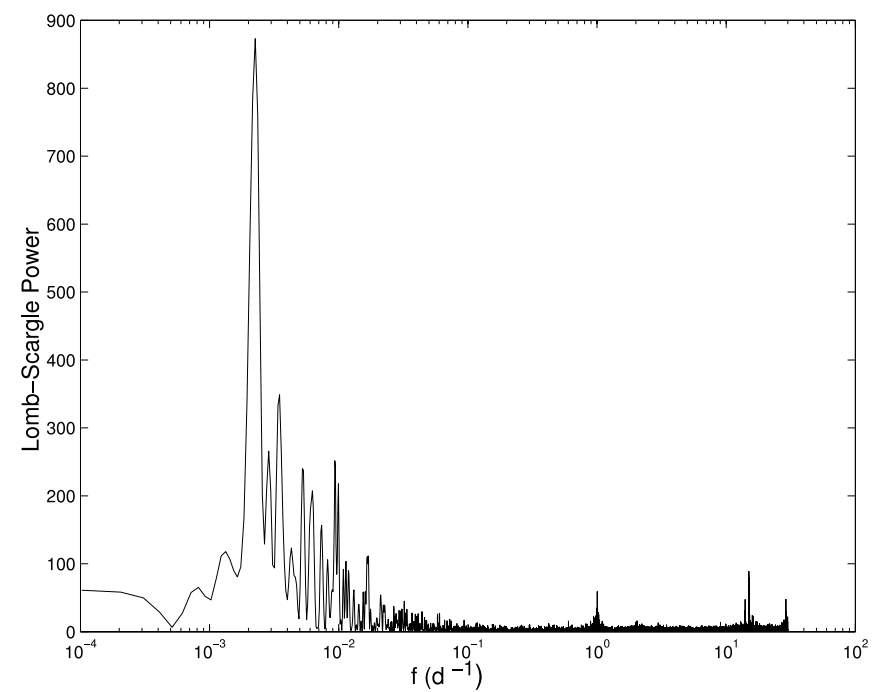

FIG. 24.-L-S periodogram for LMC X-3 in the $1.5-12 \mathrm{keV}$ band made from $8.5 \mathrm{yr}$ of $90 \mathrm{~s}$ time resolution data. Multiple peaks on timescales of $100-$ 500 days are apparent.

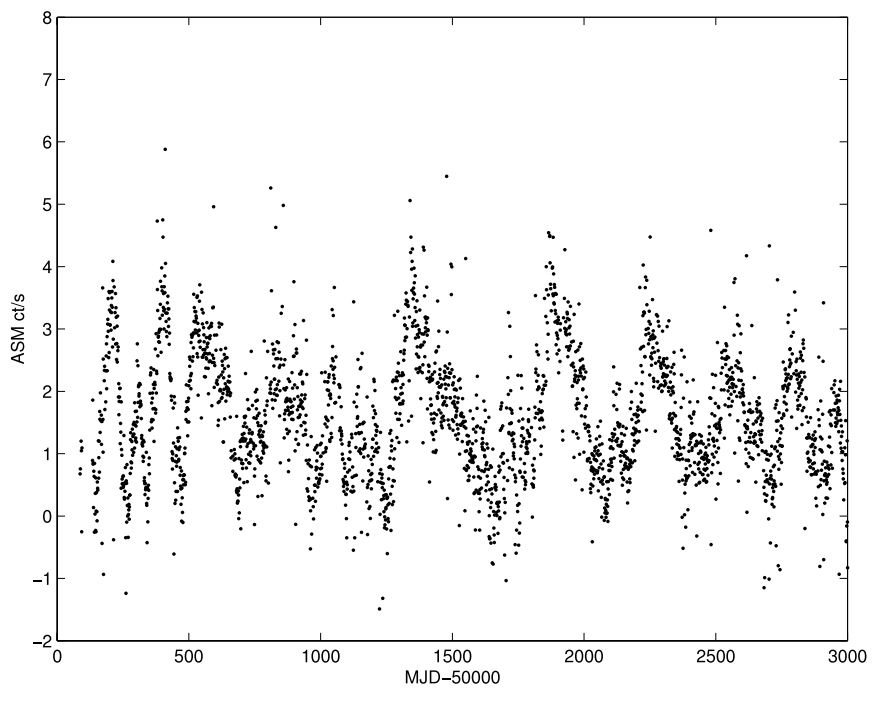

FIG. 25.-ASM 1.5-12 keV band light curve of LMC X-3. Variability on timescales of 100-500 days is apparent.

that are apparent as broad and weak features in the original L-S periodograms and therefore failed the significance test (X0115+634, X1942+274, and 4U 1145-619). We note that the two type 1 candidate periodicities only met our detection criteria in the analysis of the periodograms made from 1 day average light curves in which the threshold in terms of power (although the same in FAP) is somewhat lower than that used in the search of the higher time resolution data.

IRAS 04575-7537.- IRAS 04575-7537 is a Seyfert 2 galaxy at redshift 0.018 (Polletta et al. 1996) and Galactic latitude $b=$ $-33^{\circ}$. The detection of a 3.23 day X-ray period is based on the periodogram shown in Fig. 26. The period is detected at marginal significance in a periodogram made using data in 1 day time bins for the $1.2-15 \mathrm{keV}$ band; a peak is also visible in the periodogram for the 5-12 keV band and in a periodogram made directly using $90 \mathrm{~s}$ intensities, but it is not sufficiently high to meet our formal detection criterion. A phase-averaged light curve made by folding

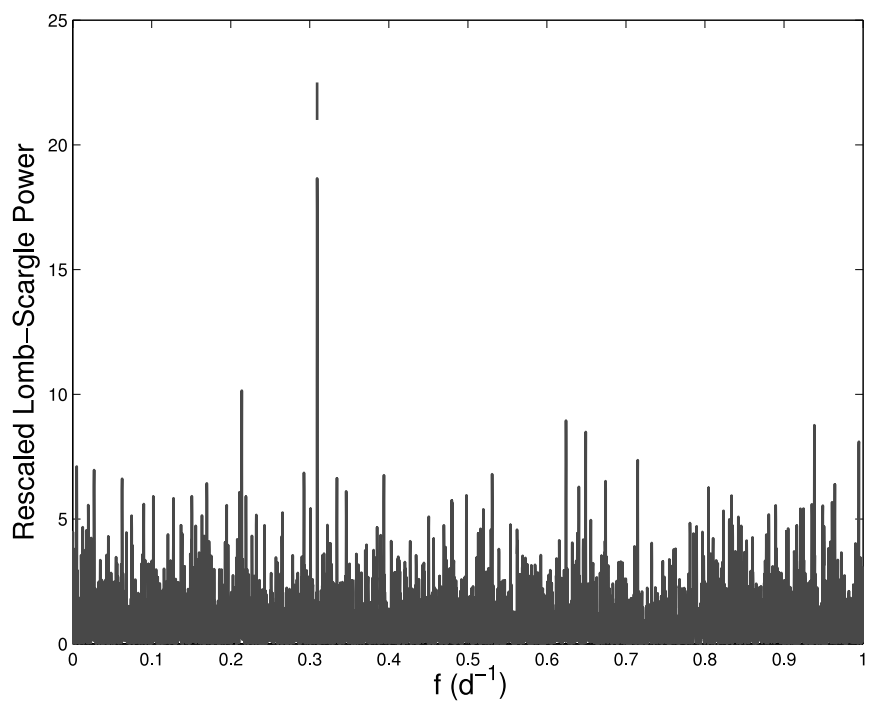

FIG. 26.- Rescaled L-S periodogram for IRAS $04575-7537$ in the 1.5$12 \mathrm{keV}$ band made from $8.5 \mathrm{yr}$ of 1 day time averages. The 3.23 day period is indicated. 


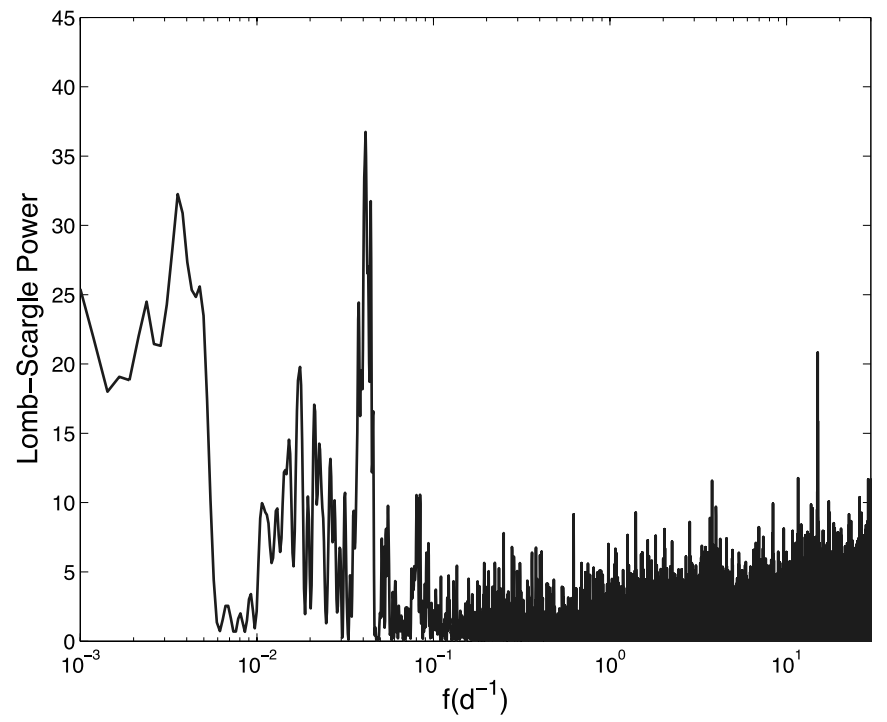

FIG. 27.-L-S periodogram for X0115-634 in the 3-5 keV band for the time interval of MJD 50,087-51,141 (before the first outburst). The 24 day known orbital period is visible.

at this period indicates a smooth modulation (see Fig. 6). Since the significance of the periodogram peak is marginal, we cannot fully exclude the possibility that it is due to a statistical fluctuation. If it is a real periodicity, it would need to result, e.g., from a foreground high Galactic latitude $\mathrm{X}$-ray binary.

$X 0115+634$ - - X0115+634 (4U 0115+634) has been long suspected to be a Be star system, and it is known to comprise a $3.6 \mathrm{~s}$ pulsar (Cominsky et al. 1978). Its optical counterpart was recently reclassified as an O9e star (Unger et al. 1998). Within the 8.5 years covered by the ASM observations, it underwent two major outbursts and some "failed" ones on intervals of $\sim 500$ days. We found that the known 24 day orbital period (Rappaport et al. 1978) appears to be the highest peak in the L-S periodogram for the first 3 yr of ASM data (before the first outbursts, earlier than MJD 51,142) (Fig. 27). However, there are broad features around

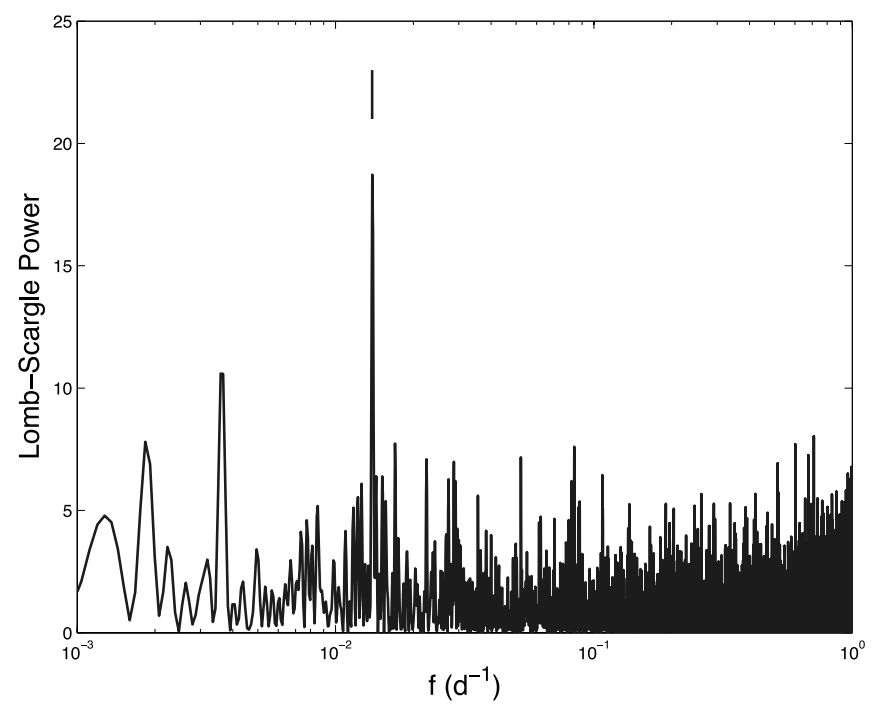

FIG. 28.-L-S periodogram for SAX J1808.4-3658 in the 3-5 keV band made from $8.5 \mathrm{yr}$ of ASM data in 1 day time bins. The $\sim 72$ day peak is indicated.

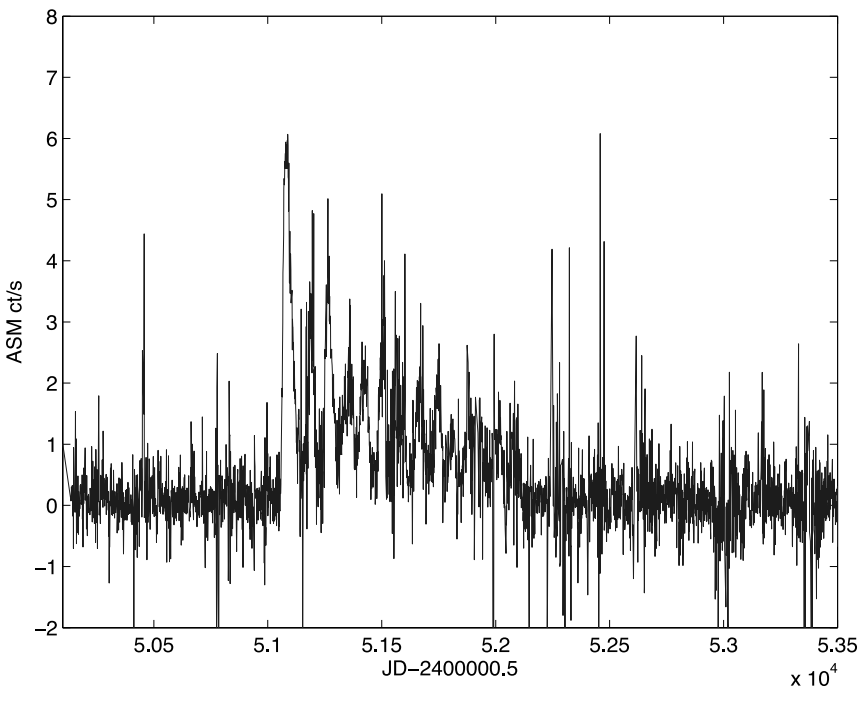

FIG. 29.-ASM light curve of X1942+274. Outbursts on timescales of 80 days are apparent for the time interval of MJD 51,000-52,000.

the period. No significant detection was made in the rescaled periodogram.

SAX J1808.4-3658.-SAX J1808.4-3658 was found by Wijnands \& van der Klis (1998) to exhibit pulsations at a frequency of $401 \mathrm{~Hz}$, which made it the first discovered accreting millisecond X-ray pulsar. A pulse timing analysis showed Doppler shifts that reveal the orbital period of about 7249 s (Chakrabarty \& Morgan 1998). In the present analysis, the periodograms of the $3-$ 5 and $1.5-3 \mathrm{keV}$ band data for all $8.5 \mathrm{yr}$ averaged in 1 day time bins show a peak at a 72 day period (Fig. 28). The 72 day period also appears as the location of the maximum power in the periodograms made using $90 \mathrm{~s}$ time bins in the $1.5-3,3-5$, and 1.5$12 \mathrm{keV}$ bands, but in these cases the strengths are not significant in terms of our detection criteria. The folded light curve (Fig. 6) appears to indicate that this detection is highly significant. However, this is misleading, because the statistics are dominated by the low-frequency behavior of the source, which is not reflected in the error bars. We find no evidence of intensity variation at the orbital period.

X1942+274. - X1942+274 (GRO J1944+26) was first discovered as a $15.8 \mathrm{~s}$ pulsar (Fishman et al. 1989). The RXTE ASM light curve of this source shows outbursts between 1998 and 2001 that recur more or less every $\sim 80$ days and may be characteristic of a Be/NS system. There was a previous report of a $\sim 80$ day period of the outbursts (Campana et al. 1999). However, the orbital period is 169.2 days (Wilson et al. 2003). The outbursts occur roughly twice per orbit at phases that are not stable. The $\sim 80$ day (quasi) periodicity is evident in the ASM light curves of X1942+274 (Fig. 29). Such periodicity also appears as the highest peak in the L-S periodogram in the $5-12 \mathrm{keV}$ band (Fig. 30), as well as the $1.5-12 \mathrm{keV}$ band. However, in the rescaled periodogram, the periodicity is not significant because of the appearance of the broad features around this period.

$4 U 1145-619$. - 4U 1145-619 is a persistent but highly variable Be/NS system. Analysis of the long-term X-ray behavior using 4 yr of Ariel 5 Sky Survey Instrument observations (Watson et al. 1981) showed four outbursts at approximately 6 month intervals. The outbursts are typically of 10 day duration, with flux levels increasing by a factor of $\sim 5$. The $7 \mathrm{yr}$ of data from Vela $5 B$ on this source are dominated by one strong outburst. In the Watson et al. paper, the power spectrum for data excluding the burst 


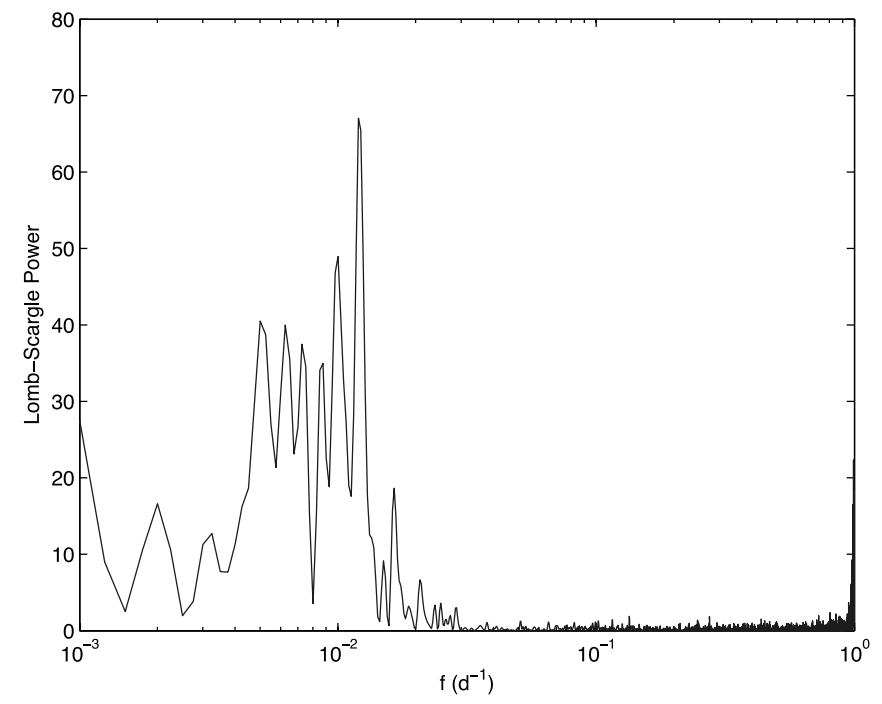

FIG. 30.-L-S periodogram for X1942+274 in the 5-12 keV band for the time interval of MJD 51,000-52,000.

showed no sign of the 187.5 day period. However, the epochfolding analysis showed some evidence for half this period (Priedhorsky \& Terrell 1983). Three separate EXOSAT observations (Warwick et al. 1985) confirmed the intensity rise and decrease, which are consistent with the ephemeris of the outbursts from previous observations.

With a 9 yr baseline, combined with reasonable sensitivity and excellent coverage, the ASM data provide the best longterm observations for this source. The ASM light curve and hardness ratios are shown in Fig. 31. At least the first three outbursts are visible in the ASM data. The times of these outbursts are consistent with the ephemeris given from previous observations (Priedhorsky \& Terrell 1983). Four other possible outbursts are also consistent with the ephemeris (the eighth, 12th,

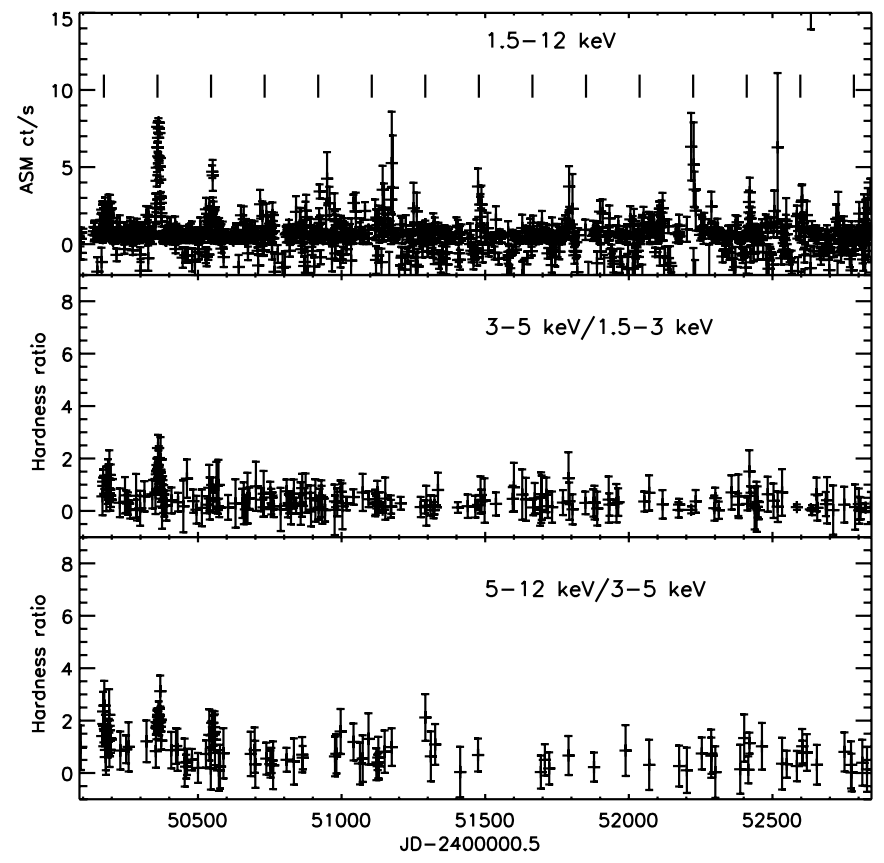

FIG. 31.-ASM light curve and hardness ratios of X1145-619. Intensity fluctuations on timescales of $\sim 188$ day intervals are labeled with short vertical lines.

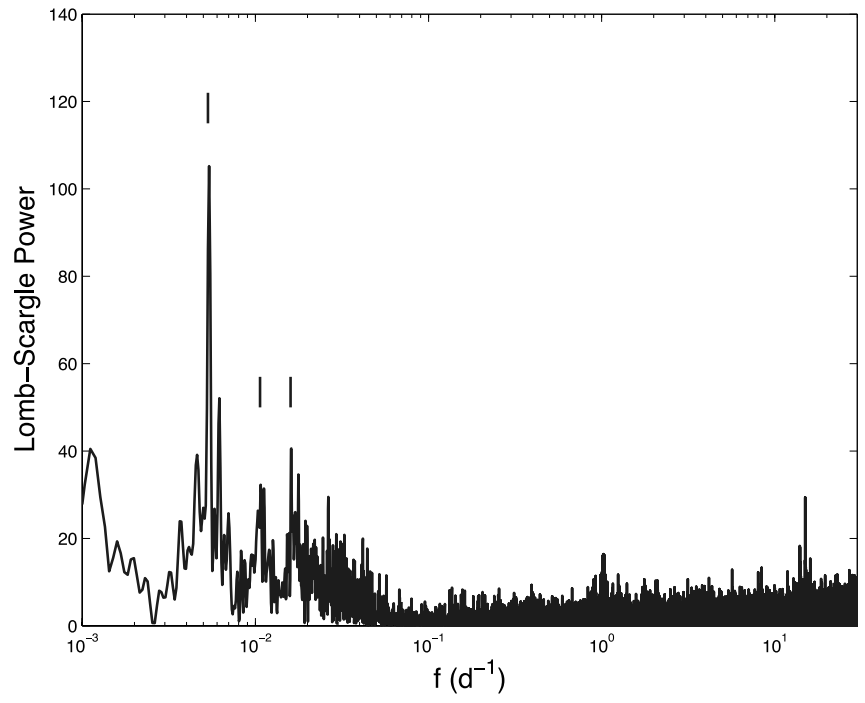

FIG. 32.-L-S periodogram of X1145-619 in the 5-12 keV band with the $8.5 \mathrm{yr}$ of ASM data in $90 \mathrm{~s}$ time bins. The $\sim 185$ day period and its harmonic are indicated with short vertical lines. There are broad structures around the period and its harmonics.

13 th, and 14th from the left). The strength of the outbursts seems to be highly variable from cycle to cycle, consistent with the Vela $5 B$ observations.

The L-S periodogram of this source for $8.5 \mathrm{yr}$ of ASM light curves is shown in Fig. 32. A peak at $187.0 \pm 3.2$ days is detected clearly. The uncertainty in the period was estimated according to the frequency bin size. Side lobes indicating modulation on a timescale of $\sim 3.3 \mathrm{yr}$ are visible.

\section{SUMMARY}

We have presented results from a systematic periodicity search through the first $8.5 \mathrm{yr}$ of source intensity measurements made using the RXTE ASM. The ASM database is an incredibly valuable resource that has allowed us to carry out this search in a uniform fashion using the intensity records for a large number of X-ray sources. Our search has served the purposes of discovering and measuring new periodicities and providing a means for improving the level of knowledge of previously known periodic phenomena in X-ray sources. The detection strategy we have adopted is conservative in general. It can, hopefully, be useful in clarifying the authenticity of some previously claimed periodicities.

The results of our search demonstrate that orbital modulation is more readily detected in HMXBs than in LMXBs (Joss \& Rappaport 1979). A total of 33 orbital periods have been securely detected in our search. Only eight of them are believed to be in LMXBs, while 24 are in HMXBs, with 16 in supergiant systems and eight in Be star systems. Of the $\sim 400 \mathrm{X}$-ray sources monitored with the ASM, more than 100 of them are believed to be LMXBs, and less than 60 are believed to be HMXBs. Yet it is apparent from the present results that there are far fewer orbital periodicities detected in the LMXBs than in the HMXBs. In particular, the fraction of eclipses observed in LMXBs is less than $3 \%$, well below that of the HMXB systems, consistent with previous observations that the number of eclipses detected in LMXBs is well below the expected rate if the companion stars are dwarf stars that fill their Roche lobe and if the accretion disks are thin. 
We gratefully acknowledge Gianluca Israel, Andrew Peele, Saul Rappaport, Ronald Remillard, and Jean Swank for helpful contributions and conversations, the efforts of the RXTE ASM groups at MIT and NASA Goddard Space Flight Center (GSFC), and the RXTE mission support groups at GSFC. We are grateful to an anonymous referee for several helpful comments. We acknowledge the support of NASA through contract NAS 5-30612 (MIT).

\section{REFERENCES}

Aleksandrovich, N. L., Aref'ev, V. A., Borozdin, K. N., Syunyaev, R. A., \& Skinner, G. K. 1995, Astron. Lett., 21, 431

Baykal, A., Inam, Ç., Ali Alpar, M., in't Zand, J., \& Strohmayer, T. 2001, MNRAS, 327, 1269

Baykal, A., Stark, M. J., \& Swank, J. 2000, ApJ, 544, L129

Blundell, K. M., Mioduszewski, A. J., Muxlow, T. W. B., Podsiadlowski, P., \& Rupen, M. P. 2001, ApJ, 562, L79

Boyd, P. T., \& Smale, A. P. 2004, ApJ, 612, 1006

Bradt, H. V., Levine, A. M., Remillard, R. A., \& Smith, D. A. 2000, in Rossi 2000: Astrophysics with the Rossi X-Ray Timing Explorer (Greenbelt: NASA), E114

Callanan, P. J., Grindlay, J. E., \& Cool, A. M. 1995, PASJ, 47, 153

Campana, S., Israel, G., \& Stella, L. 1999, A\&A, 352, L91

Casares, J., Charles, P., \& Kuulkers, E. 1998, ApJ, 493, L39

Chakrabarty, D., \& Morgan, E. H. 1998, Nature, 394, 346

Chou, Y., Grindlay, J. E., \& Bloser, P. F. 2001, ApJ, 549, 1135

Church, M. J., Dotani, T., Bałucińska-Church, M., Mitsuda, K., Takahashi, T., Inoue, H., \& Yoshida, K. 1997, ApJ, 491, 388

Clarkson, W. I., Charles, P. A., Coe, M. J., Laycock, S., Tout, M. D., \& Wilson, C. A. 2003, MNRAS, 339, 447

Cominsky, L., Clark, G. W., Li, F., Mayer, W., \& Rappaport, S. 1978, Nature, 273, 367

Cominsky, L. R., \& Wood, K. S. 1989, ApJ, 337, 485

Corbet, R., Peele, A., \& Remillard, R. 1997, IAU Circ., 6556, 3

Corbet, R. H. D. 2003, ApJ, 595, 1086

Corbet, R. H. D., Finley, J. P., \& Peele, A. G. 1999a, ApJ, 511, 876

Corbet, R. H. D., Hannikainen, D. C., \& Remillard, R. 2004, ATel, 269

Corbet, R. H. D., Marshall, F. E., Peele, A. G., \& Takeshima, T. 1999b, ApJ, 517,956

Corbet, R. H. D., \& Mukai, K. 2002, ApJ, 577, 923

Corbet, R. H. D., \& Peele, A. G. 1997, ApJ, 489, L83

. 2000, ApJ, 530, L33

. 2001, ApJ, 562, 936

Corbet, R. H. D., \& Remillard, R. 2005, ATel, 377

Cowley, A. P., Crampton, D., \& Hutchings, J. B. 1979, ApJ, 231, 539

Cowley, A. P., et al. 1991, ApJ, 381, 526

Cox, N. L. J., Kaper, L., \& Mokiem, M. R. 2005, A\&A, 436, 661

Crampton, D., Cowley, A. P., \& Hutchings, J. B. 1980, ApJ, 235, L131

Crampton, D., Hutchings, J. B., \& Cowley, A. P. 1985, ApJ, 299, 839

Delgado-Martí, H., Levine, A. M., Pfahl, E., \& Rappaport, S. A. 2001, ApJ, 546,455

den Hartog, P. R., Kuiper, L. M., Corbet, R. H. D., in 't Zand, J. J. M., Hermsen, W., Vink, J., Remillard, R., \& van der Klis, M. 2004, ATel, 281

Dieters, S., O’Neill, P., Farrell, S., \& Sood, R. 2005, Adv. Space Res., 35, 1185

Eikenberry, S. S., Cameron, P. B., Fierce, B. W., Kull, D. M., Dror, D. H., Houck, J. R., \& Margon, B. 2001, ApJ, 561, 1027

Farrell, S., Sood, R., \& O'Neill, P. 2004, ATel, 283

Farrell, S. A., O’Neill, P. M., \& Sood, R. K. 2005, Publ. Astron. Soc. Australia, 22, 267

Finley, J. P., Belloni, T., \& Cassinelli, J. P. 1992, A\&A, 262, L25

Finley, J. P., Taylor, M., \& Belloni, T. 1994, ApJ, 429, 356

Fishman, G. J., et al. 1989, in Proc. GRO Science Workshop, ed. W. N. Johnson (Greenbelt: NASA), 2

Gies, D. R., McSwain, M. V., Riddle, R. L., Wang, Z., Wiita, P. J., \& Wingert, D. W. 2002, ApJ, 566, 1069

Grebenev, S. A., Ubertini, P., Chenevez, J., Mowlavi, N., Roques, J.-P., Gehrels, N., \& Kuulkers, E. 2004, ATel, 350

Grindlay, J., Gursky, H., Schnopper, H., Parsignault, D. R., Heise, J., Brinkman, A. C., \& Schrijver, J. 1976, ApJ, 205, L127

Gruber, D. E., \& Rothschild, R. E. 1984, ApJ, 283, 546

Hall, T. A., Finley, J. P., Corbet, R. H. D., \& Thomas, R. C. 2000, ApJ, 536, 450

Hannikainen, D. C., Rodriguez, J., Cabanac, C., Schultz, J., Lund, N., Vilhu, O.,

Petrucci, P. O., \& Henri, G. 2004, A\&A, 423, L17

Horne, J. H., \& Baliunas, S. L. 1986, ApJ, 302, 757

Hulleman, F., in 't Zand, J. J. M., \& Heise, J. 1998, A\&A, 337, L25

Ilovaisky, S. A., Aurière, M., Koch-Miramond, L., Chevalier, C., Cordoni, J.-P., \& Crowe, R. A. 1993, A\&A, 270, 139

in 't Zand, J., \& Heise, J. 2004, ATel, 362

in 't Zand, J. J. M., et al. 2000, A\&A, 355, 145

2003, A\&A, 406, 233
Israel, G. L., \& Stella, L. 1996, ApJ, 468, 369

Joss, P. C., \& Rappaport, S. 1979, A\&A, 71, 217

Katz, J. L. 1973, Nature Phys. Sci., 246, 87

Kotani, T., Kawai, N., Matsuoka, M., \& Brinkmann, W. 1996, PASJ, 48, 619

Lasota, J.-P. 2001, NewA Rev., 45, 449

Levine, A., Rappaport, S., Deeter, J. E., Boynton, P. E., \& Nagase, F. 1993 , ApJ, 410, 328

Levine, A. M., Bradt, H., Cui, W., Jernigan, J. G., Morgan, E. H., Remillard, R., Shirey, R. E., \& Smith, D. A. 1996, ApJ, 469, L33

Levine, A. M., \& Jernigan, J. G. 1982, ApJ, 262, 294

Levine, A. M., Rappaport, S., Remillard, A., \& Savcheva, A. 2004, ApJ, 617, 1284

Liang, E. P., \& Nolan, P. L. 1984, Space Sci. Rev., 38, 353

Lomb, N. R. 1976, Ap\&SS, 39, 447

Margon, B. 1984, ARA\&A, 22, 507

Marshall, H. L., Canizares, C. R., \& Schulz, N. S. 2002, ApJ, 564, 941

Mason, K. O., Middleditch, J., Nelson, J. E., White, N. E., Seitzer, P., Tuohy, I. R., \& Hunt, L. K. 1980, ApJ, 242, L109

Migliari, S., Fender, R., \& Méndez, M. 2002, Science, 297, 1673

Milgrom, M. 1978, A\&A, 67, L25

Morel, T., \& Grosdidier, Y. 2005, MNRAS, 356, 665

Negueruela, I., \& Reig, P. 2001, A\&A, 371, 1056

Negueruela, I., Roche, P., Buckley, D. A. H., Chakrabarty, D., Coe, M. J., Fabregat, J., \& Reig, P. 1996, A\&A, 315, 160

Oda, M. 1977, Space Sci. Rev., 20, 757

Paredes, J. M., Martí, J., Peracaula, M., \& Ribó, M. 1997, A\&A, 320, L25

Parmar, A. N., Smale, A. P., Verbunt, F., \& Corbet, R. H. D. 1991, ApJ, 366, 253

Parmar, A. N., White, N. E., Giommi, P., \& Gottwald, M. 1986, ApJ, 308, 199 Paul, B., Kitamoto, S., \& Makino, F. 2000, ApJ, 528, 410

Polletta, M., Bassani, L., Malaguti, G., Palumbo, G. G. C., \& Caroli, E. 1996, ApJS, 106, 399

Press, W. H., Teukolsky, S. A., Vetterling, W. T., \& Flannery, B. P. 1992, Numerical Recipes in C (2nd ed.; Cambridge: Cambridge Univ. Press)

Priedhorsky, W. C., Brandt, S., \& Lund, N. 1995, A\&A, 300, 415

Priedhorsky, W. C., \& Terrell, J. 1983, ApJ, 273, 709

1984a, ApJ, 284, L17

- 1984b, ApJ, 280, 661

. 1986, ApJ, 301, 886

Pringle, J. E. 1996, MNRAS, 281, 357

Rappaport, S., Clark, G. W., Cominsky, L., Joss, P. C., \& Li, F. 1978, ApJ, 224, L1

Reig, P., Chakrabarty, D., Coe, M. J., Fabregat, J., Negueruela, I., Prince, T. A., Roche, P., \& Steele, I. A. 1996, A\&A, 311, 879

Reig, P., Negueruela, I., Fabregat, J., Chato, R., Blay, P., \& Mavromatakis, F. 2004, A\&A, 421, 673

Reig, P., Negueruela, I., Papamastorakis, G., Manousakis, A., \& Kougentakis, T. 2005, A\&A, 440, 637

Reig, P., \& Roche, P. 1999, MNRAS, 306, 95

Saraswat, P., \& Apparao, K. M. V. 1992, ApJ, 401, 678

Scargle, J. D. 1982, ApJ, 263, 835

Schreier, E., Giacconi, R., Gursky, H., Kellogg, E., \& Tananbaum, H. 1972, ApJ, 178, L71

Smale, A. P., Church, M. J., \& Bałucińska-Church, M. 2001, ApJ, 550, 962

Smale, A. P., \& Lochner, J. C. 1992, ApJ, 395, 582

Smale, A. P., Mason, K. O., Williams, O. R., \& Watson, M. G. 1989, PASJ, 41, 607

Steiner, J. E., Ferrara, A., Garcia, M., Patterson, J., Schwartz, D. A., Warwick, R. S., Watson, M. G., \& McClintock, J. E. 1984, ApJ, 280, 688

Stella, L., Priedhorsky, W., \& White, N. E. 1987, ApJ, 312, L17

Swank, J. H., \& Markwardt, C. B. 2003, ATel, 128 2004, ATel, 359

Torrejón, J. M., \& Negueruela, I. 2004, ATel, 370

Unger, S. J., Roche, P., Negueruela, I., Ringwald, F. A., Lloyd, C., \& Coe, M. J. 1998, A\&A, 336, 960

Verbunt, F., \& van den Heuvel, E. P. J. 1995, in X-Ray Binaries, ed. W. G. H. Lewin, J. van Paradijs, \& E. P. J. van den Heuvel (Cambridge: Cambridge Univ. Press), 457

Wachter, S., Smale, A. P., \& Bailyn, C. 2000, ApJ, 534, 367 
Walter, F. M., Bowyer, S., Mason, K. O., Clarke, J. T., Henry, J. P., Halpern, J., \& Grindlay, J. E. 1982, ApJ, 253, L67

Warwick, R. S., Watson, M. G., \& Willingale, R. 1985, Space Sci. Rev., 40, 429

Watson, M. G., Warwick, R. S., \& Ricketts, M. J. 1981, MNRAS, 195, 197

Watson, M. G., Willingale, R., King, A. R., Grindlay, J. E., \& Halpern, J. 1985, Space Sci. Rev., 40, 195

Wen, L. 2001, Ph.D. thesis, MIT

Wen, L., Cui, W., Levine, A. M., \& Bradt, H. V. 1999, ApJ, 525, 968

Wen, L., Remillard, R. A., \& Bradt, H. V. 2000, ApJ, 532, 1119

White, N. E., Mason, K. O., Sanford, P. W., \& Murdin, P. 1976, MNRAS, 176, 201
White, N. E., Nagase, F., \& Parmar, A. N. 1995, in X-Ray Binaries, ed. W. G. H. Lewin, J. van Paradijs, \& E. P. J. van den Heuvel (Cambridge: Cambridge Univ. Press), 1

White, N. E., \& Swank, J. H. 1982, ApJ, 253, L61

Wijnands, R., \& van der Klis, M. 1998, Nature, 394, 344

Wilson, C. A., Finger, M. H., Coe, M. J., \& Negueruela, I. 2003, ApJ, 584, 996 Wilson, C. A., Finger, M. H., Harmon, B. A., Chakrabarty, D., \& Strohmayer, T. 1998, ApJ, 499, 820

Wojdowski, P., Clark, G. W., Levine, A. M., Woo, J. W., \& Zhang, S. N. 1998, ApJ, 502, 253

Yoshida, K. 1993, Ph.D. thesis, Kanagawa Univ. 